\title{
Bounded languages described by GF(2)-grammars
}

\author{
Vladislav Makarov*
}

November 22, 2021

\begin{abstract}
GF(2)-grammars are a recently introduced grammar family that have some unusual algebraic properties and are closely connected to unambiguous grammars. By using the method of formal power series, we establish strong conditions that are necessary for subsets of $a^{*} b^{*}$ and $a^{*} b^{*} c^{*}$ to be described by some GF(2)-grammar. By further applying the established results, we settle the long-standing open question of proving the inherent ambiguity of the language $\left\{a^{n} b^{m} c^{\ell} \mid n \neq m\right.$ or $\left.m \neq \ell\right\}$, as well as give a new, purely algebraic, proof of the inherent ambiguity of the language $\left\{a^{n} b^{m} c^{\ell} \mid n=m\right.$ or $\left.m=\ell\right\}$.
\end{abstract}

Keywords: Formal grammars, finite fields, bounded languages, unambiguous grammars, inherent ambiguity.

\section{Introduction}

GF(2)-grammars, recently introduced by Bakinova et al. [3], and further studied by Makarov and Okhotin [15], are a variant of ordinary context-free grammars, in which the disjunction is replaced by exclusive OR, whereas the classical concatenation is replaced by a new operation called GF(2)-concatenation: $K \odot L$ is the set of all strings with an odd number of partitions into a concatenation of a string in $K$ and a string in $L$.

There are several motivating reasons behind studying GF(2)-grammars. The first of them they are a class of grammars with better algebraic properties, compared to ordinary grammars and similar grammar families, because the underlying boolean semiring logic was replaced by a logic of a field with two elements. As we will see later in the paper, that makes GF(2)-grammars lend themselves very well to algebraic manipulations.

The second reason is that GF(2)-grammars provide a new way of looking at unambiguous grammars. For example, instead of proving that some language is inherently ambiguous, one can prove that no $\mathrm{GF}(2)$-grammar describes it. While the latter condition is, strictly speaking, stronger, it may turn out to be easier to prove, because GF(2)-grammars have good algebraic properties and are also closed under symmetric difference, meaning that more tools can be used in the proof.

\footnotetext{
*Saint-Petersburg State University. Supported by Russian Science Foundation, project 18-11-00100.
} 
Finally, GF(2)-grammars generalize the notion of parity nondeterminism to grammars. Recall that the most common types of nondeterminism that are considered in complexity theory are classical nondeterminism, which corresponds to an existence of an accepting computation, unambiguous nondeterminism, which corresponds to an existence of unique accepting computation and parity nondeterminism, which corresponds to number of accepting computations being odd.

Parity complexity classes, mainly $\oplus L$ and $\oplus P$, are actively studied in the complexity theory. Perhaps most famously, the latter is used in the proof of Toda's theorem, which statement does not concern any parity classes directly.

Similarly to the parity complexity classes, the notion of $\oplus$ FA was considered before [17], as a counterpart to NFA and UFA (unambiguous finite automaton).

Tying to the theme of connections between unambiguous and GF(2)-grammars, classical and parity nondeterminism can be seen as two different generalisations of unambiguous nondeterminism: if the number of accepting computations is 0 or 1 , then it is positive (classical case) and odd (parity case) at the same time; the same is not true for larger numbers, of course.

The main result of this paper is Theorem 4.2 that establishes a strong necessary conditions on subsets of $a_{1}^{*} a_{2}^{*} \ldots a_{k}^{*}$ that are described by GF(2)-grammars. Theorem 4.1, a special case of Theorem 4.2, implies that there are no $\mathrm{GF}(2)$-grammars for the languages $L_{1}:=\left\{a^{n} b^{m} c^{\ell} \mid n=m\right.$ or $\left.m=\ell\right\}$ and $L_{2}:=\left\{a^{n} b^{m} c^{\ell} \mid n \neq m\right.$ or $\left.m \neq \ell\right\}$.

As a consequence, both are inherently ambiguous. For $L_{1}$, all previously known arguments establishing its inherent ambiguity were combinatorial, mainly based on Ogden's lemma.

Proving inherent ambiguity of $L_{2}$ was a long-standing open question due to Autebert et al. [2, p. 375]. There is an interesting detiail here: back in 1966, Ginsburg and Ullian fully characterized bounded languages described by unambiguous grammars in terms of semi-linear sets [12, Theorems 5.1 and 6.1]. However, most natural ways to apply this characterization suffer from the same limitation: they mainly rely on words that are not in the language and much less on the words that are. Hence, "dense" languages like $L_{2}$ leave them with almost nothing to work with. Moreover, $L_{2}$ has an algebraic generating function, meaning that analytic methods cannot tackle it either. In fact, Flajolet [6], in his seminal work on analytic methods for proving grammar ambiguity, refers to inherent ambiguity of $L_{2}$ as to a still open question (see page 286).

\section{Basics}

The proofs that we will see later make heavy use of algebraic methods. For the algebraic parts, the exposition strives to be as elementary and self-contained as possible. Hence, I will prove a lot of lemmas that are by no way original and may be considered trivial by someone with good knowledge of commutative algebra. This is the intended effect; if you consider something to be trivial, you can skip reading the proof. If, on the other hand, you have some basic knowledge of algebra, but still find some of the parts to be unclear, you may 
contact me and I will try to find a better wording. The intended "theoretical minimum" is being at least somewhat familiar with concepts of polynomials, rational functions and formal power series.

But let us recall the definition and the basic properties of GF(2)-grammars first. This section is completely based on already published work: the original paper about $\mathrm{GF}(2)$ operations by Bakinova et al. [3] and the paper about basic properties of GF(2)-grammars by Makarov and Okhotin [15]. Hence, all the proofs are omitted; for proofs and more thorough commentary on definitions refer to the aforementioned papers. If you are already familiar with both of them, you may skip straight to the next section.

GF(2)-grammars are built upon GF(2)-operations [3]: symmetric difference and new operation called $\mathrm{GF}(2)$-concatenation:

$K \odot L=\{w \mid$ the number of partitions $w=u v$, with $u \in K$ and $v \in L$, is odd $\}$

From a syntactical standpoint, GF(2)-grammars do not differ from ordinary grammars. However, in the right-hand sides of the rules, the normal concatenation is replaced with $\mathrm{GF}(2)$-concatenation, whereas multiple rules for the same nonterminal correspond to symmetric difference of given conditions, instead of their disjunction.

Definition ([3]). A GF(2)-grammar is a quadruple $G=(\Sigma, N, R, S)$, where:

- $\Sigma$ is the alphabet of the language;

- $N$ is the set of nonterminal symbols;

- every rule in $R$ is of the form $A \rightarrow X_{1} \odot \ldots \odot X_{\ell}$, with $\ell \geqslant 0$ and $X_{1}, \ldots X_{\ell} \in \Sigma \cup N$, which represents all strings that have an odd number of partitions into $w_{1} \ldots w_{\ell}$, with each $w_{i}$ representable as $X_{i}$;

- $S \in N$ is the initial symbol.

The grammar must satisfy the following condition. Let $\widehat{G}=(\Sigma, N, \widehat{R}, S)$ be the corresponding ordinary grammar, with $\widehat{R}=\left\{A \rightarrow X_{1} \ldots X_{\ell} \mid A \rightarrow X_{1} \odot \ldots \odot X_{\ell} \in R\right\}$. It is assumed that, for every string $w \in \Sigma^{*}$, the number of parse trees of $w$ in $\widehat{G}$ is finite; if this is not the case, then $G$ is considered ill-formed.

Then, for each $A \in N$, the language $L_{G}(A)$ is defined as the set of all strings with an odd number of parse trees as $A$ in $\widehat{G}$.

Theorem A ([3]). Let $G=(\Sigma, N, R, S)$ be a GF(2)-grammar. Then the substitution $A=L_{G}(A)$ for all $A \in N$ is a solution of the following system of language equations.

$$
A=\bigsqcup_{A \rightarrow X_{1} \odot \ldots \odot X_{\ell} \in R} X_{1} \odot \ldots \odot X_{\ell} \quad(A \in N)
$$

Multiple rules for the same nonterminal symbol can be denoted by separating the alternatives with the "sum modulo two" symbol $(\oplus)$, as in the following example. 
Example 2.1 ([3]). The following GF(2)-linear grammar defines the language $\left\{a^{\ell} b^{m} c^{n} \mid\right.$ $\ell=m$ or $m=n$, but not both $\}$.

$$
\begin{aligned}
& S \rightarrow A \oplus C \\
& A \rightarrow a A \oplus B \\
& B \rightarrow b B c \oplus \epsilon \\
& C \rightarrow C c \oplus D \\
& D \rightarrow a D b \oplus \epsilon
\end{aligned}
$$

Indeed, each string $a^{\ell} b^{m} c^{n}$ with $\ell=m$ or with $m=n$ has a parse tree, and if both equalities hold, then there are accordingly two parse trees, which cancel each other.

Example 2.2 ([3]). The following grammar describes the language $\left\{a^{2^{n}} \mid n \geqslant 0\right\}$.

$$
S \rightarrow(S \odot S) \oplus a
$$

The main idea behind this grammar is that the GF(2)-square $S \odot S$ over a unary alphabet doubles the length of each string: $L \odot L=\left\{a^{2 \ell} \mid a^{\ell} \in L\right\}$. The grammar iterates this doubling to produce all powers of two.

As the previous example illustrates, GF(2)-grammars can describe non-regular unary languages, unlike ordinary grammars. We will need the classification of unary languages describable by GF(2)-grammars in the following Sections.

Definition. A set of natural numbers $S \subseteq \mathbb{N}$ is called q-automatic [1], if there is a finite automaton over the alphabet $\Sigma_{q}=\{0,1, \ldots, q-1\}$ recognizing base- $q$ representations of these numbers.

Let $\mathbb{F}_{q}[t]$ be the ring of polynomials over the $q$-element field $\operatorname{GF}(q)$, and let $\mathbb{F}_{q}[[t]]$ denote the ring of formal power series over the same field.

Definition. A formal power series $f \in \mathbb{F}_{q}[[t]]$ is said to be algebraic, if there exists a non-zero polynomial $P$ with coefficients from $\mathbb{F}_{q}[t]$, such that $P(f)=0$.

Theorem B (Christol's theorem for GF(2) [7]). A formal power series $\sum_{n=0}^{\infty} f_{n} t^{n} \in \mathbb{F}_{2}[[t]]$ is algebraic if and only if the set $\left\{n \in \mathbb{N}_{0} \mid f_{n}=1\right\}$ is 2-automatic.

Theorem C (Unary languages described by GF(2)-grammars [15]). For a unary alphabet, the class of all 2-automatic languages coincides with the class of all languages described by GF(2)-grammars.

\section{Subsets of $a^{*} b^{*}$}

Suppose that some GF(2)-grammar over an alphabet $\Sigma=\{a, b\}$ generates a language that is a subset of $a^{*} b^{*}$. How does the resulting language look like? 
It will prove convenient to associate subsets of $a^{*} b^{*}$ with (commutative) formal power series in two variables $a$ and $b$ over the field $\mathbb{F}_{2}$. This correspondence is similar to the correspondence between languages over a unary alphabet with $\operatorname{GF}(2)$-operations $(\odot, \triangle)$ and formal power series of one variable with multiplication and addition [15].

Formally speaking, for every set $S \subset \mathbb{N}_{0}^{2}$, the language $\left\{a^{n} b^{m} \mid(n, m) \in S\right\} \subset a^{*} b^{*}$ corresponds to the formal power series $\sum_{(n, m) \in S} a^{n} b^{m}$ in variables $a$ and $b$. Let us denote this correspondence by asSeries: $2^{a^{*} b^{*}} \rightarrow \mathbb{F}_{2}[[a, b]]$. Then, $\operatorname{asSeries}(L \triangle K)=\operatorname{asSeries}(L)+$ asSeries $(K)$, so symmetric differences of languages corresponds to addition of power series.

On the other hand, multiplication of formal power series does not always correspond to the GF(2)-concatenation of languages. Indeed, $\operatorname{GF}(2)$-concatenation of subsets of $a^{*} b^{*}$ does not have to be a subset of $a^{*} b^{*}$. However, the correspondence does hold in the following important special case.

Lemma 3.1. If $K \subset a^{*}$ and $L \subset a^{*} b^{*}$, then $\operatorname{asSeries}(K \odot L)=\operatorname{asSeries}(K) \cdot \operatorname{asSeries}(L)$. The same conclusion holds when $K \subset a^{*} b^{*}$ and $L \subset b^{*}$.

Sketch of the proof. Follows from definitions.

Denote the set of all algebraic power series from $\mathbb{F}_{2}[[a]]$ by $\mathcal{A}$. By Christol's theorem [7], the set $\mathcal{A}$ coresponds to the set of all 2-automatic languages over an alphabet $\{a\}$. Similarly, denote the set of all algebraic power series from $\mathbb{F}_{2}[[b]]$ by $\mathcal{B}$.

Denote the set $\mathbb{F}_{2}[a, b]$ of all polynomials in variables $a$ and $b$ by poly $(a, b)$ and the set $\mathbb{F}_{2}(a, b)$ of all rational functions in variables $a$ and $b$ by $\operatorname{rat}(a, b)$. It should be mentioned that poly $(a, b)$ is a subset $\mathbb{F}_{2}[[a, b]]$, but $\operatorname{rat}(a, b)$ is not. Indeed, $\frac{1}{a} \in \operatorname{rat}(a, b)$, but not in $\mathbb{F}_{2}[[a, b]]$. The following statement is true: $\operatorname{rat}(a, b) \subset \mathbb{F}_{2}((a, b))$, where $\mathbb{F}_{2}((a, b))$ denotes the set of all Laurent series in variables $a$ and $b$. Laurent series are defined as the fractions of formal power series with equality, addition and multiplication defined in the usual way.

We will allow Laurent series to appear in intermediate results, because intermediate calculations require division, and formal power series are not closed under division. However, there are no Laurent series in the statements of the main theorem, because they do not correspond to valid languages, unless they are valid formal power series as well.

From now on, there are two possible roads this proof can take: the original argument that is more elementary, but requires lengthy manipulations with what I called algebraic expressions, and a more abstract, but much simpler approach suggested by an anonymous reviewer from MFCS 2020 conference, relying on well-known properties of field extensions. The main body of the paper follows the latter approach. The former approach can be found in the appendix.

Definition. Denote by $R_{a, b}$ the set of all Laurent series that can be represented as $\frac{\sum_{i=1}^{n} A_{i} B_{i}}{p}$, where $n$ is a nonnegative integer, $A_{i} \in \mathcal{A}$ and $B_{i} \in \mathcal{B}$ for all $i$ from 1 to $n$, and $p \in$ $\operatorname{poly}(a, b), p \neq 0$.

It is not hard to see that $R_{a, b}$ is a commutative ring. However, a even stronger statement is true: 
Lemma 3.2. $R_{a, b}$ is a field.

Proof outline. $R_{a, b}$ is the result of adjoining the elements of $\mathcal{A} \cup \mathcal{B}$, which are all algebraic over $\operatorname{rat}(a, b)$, to $\operatorname{rat}(a, b)$. It is known that the result of adjoining an arbitrary set of algebraic elements to a field is a larger field.

\subsection{The main result for subsets of $a^{*} b^{*}$}

Let us establish our main result about subsets of $a^{*} b^{*}$.

Theorem 3.1. Assume that a language $K \subset a^{*} b^{*}$ is described by a $G F(2)$-grammar. Then, the corresponding power series $\operatorname{asSeries}(K)$ is in the set $R_{a, b}$.

Proof. Without loss of generality, the GF(2)-grammar that describes $K$ is in the Chomsky normal form [3, Theorem 5]. Moreover, we can assume that $K$ does not contain an empty string.

The language $a^{*} b^{*}$ is accepted by the following DFA $M: M$ has two states $q_{a}$ and $q_{b}$, both accepting, and its transition function is $\delta\left(q_{a}, a\right)=q_{a}, \delta\left(q_{a}, b\right)=q_{b}, \delta\left(q_{b}, b\right)=q_{b}$.

Let us formally intersect the $\mathrm{GF}(2)$-grammar $G$ with regular language $a^{*} b^{*}$, recognized by the automaton $M$ (the construction of intersection of an ordinary grammar with regular expression by Bar-Hilal et al. [4] can be easily adapted to the case of GF(2)-grammars [15, Section 6]). The language described by the GF(2)-grammar will not change, because it already was a subset of $a^{*} b^{*}$ before.

The grammar itself changes considerably, however. Every nonterminal $C$ of the original $\mathrm{GF}$ (2)-grammar splits into three nonterminals: $C_{a \rightarrow a}, C_{a \rightarrow b}$. and $C_{b \rightarrow b}$. These nonterminals will satisfy the following conditions: $L\left(C_{a \rightarrow a}\right)=L(C) \cap a^{*}, L\left(C_{b \rightarrow b}\right)=L(C) \cap b^{*}$ and $L\left(C_{a \rightarrow b}\right)=L(C) \cap\left(a^{*} b^{+}\right)$. Also, a new starting nonterminal $S^{\prime}$ appears.

Moreover, every "normal" rule $C \rightarrow D E$ splits into four rules: $C_{a \rightarrow a} \rightarrow D_{a \rightarrow a} E_{a \rightarrow a}$, $C_{a \rightarrow b} \rightarrow D_{a \rightarrow a} E_{a \rightarrow b}, C_{a \rightarrow b} \rightarrow D_{a \rightarrow b} E_{b \rightarrow b}$ and $C_{b \rightarrow b} \rightarrow D_{b \rightarrow b} E_{b \rightarrow b}$.

The following happens with "final" rules: $C \rightarrow b$ turns into two rules $C_{a \rightarrow b} \rightarrow b$ and $C_{b \rightarrow b} \rightarrow b$, and $C \rightarrow a$ turns into one rule $C_{a \rightarrow a} \rightarrow a$. Finally, two more rules appear: $S^{\prime} \rightarrow S_{a \rightarrow a}$ and $S^{\prime} \rightarrow S_{a \rightarrow b}$.

For every nonterminal $C$ of the original GF(2)-grammar, the languages $L\left(C_{a \rightarrow a}\right)$ and $L\left(C_{b \rightarrow b}\right)$ are 2-automatic languages over unary alphabets $\{a\}$ and $\{b\}$ respectively. Indeed, every parse tree of $C_{a \rightarrow a}$ contains only nonterminals of type $a \rightarrow a$. Therefore, only symbol $a$ can occur as a terminal in the parse tree. So, $L\left(C_{a \rightarrow a}\right)$ is described by some $\mathrm{GF}(2)$-grammar over an alphabet $\{a\}$, and is therefore 2-automatic.

By Theorem A, the languages $L\left(C_{a \rightarrow b}\right)$ for each nonterminal $C_{a \rightarrow b}$ of the new grammar satisfy the following system of language equations (System (1)).

Here, for each nonterminal $C$, the summation is over all rules $C \rightarrow D E$ of the original $\operatorname{GF}(2)$-grammar. Also, end $\left(C_{a \rightarrow b}\right)$ is either $\{b\}$ or $\varnothing$, depending on whether or not there is a rule $C_{a \rightarrow b} \rightarrow b$ in the new $\mathrm{GF}(2)$-grammar.

$$
L\left(C_{a \rightarrow b}\right)=\operatorname{end}\left(C_{a \rightarrow b}\right) \oplus \bigoplus_{(C \rightarrow D E) \in R}\left(L\left(D_{a \rightarrow a}\right) \odot L\left(E_{a \rightarrow b}\right)\right) \oplus\left(L\left(D_{a \rightarrow b}\right) \odot L\left(E_{b \rightarrow b}\right)\right)
$$


It is easy to see that all $\mathrm{GF}(2)$-concatenations in the right-hand sides satisfy the conditions of Lemma 3.1. Denote $\operatorname{asSeries}\left(L\left(C_{a \rightarrow b}\right)\right)$ by $\operatorname{Center}(C)$, asSeries $\left(L\left(C_{a \rightarrow a}\right)\right)$ by $\operatorname{Left}(C)$, asSeries $\left(L\left(C_{b \rightarrow b}\right)\right)$ by $\operatorname{Right}(C)$ and asSeries $\left(\operatorname{end}\left(C_{a \rightarrow b}\right)\right)$ by $\operatorname{final}(C)$ for brevity. Therefore, the algebraic equivalent of System (1) also holds:

$$
\operatorname{Center}(C)=\operatorname{final}(C)+\sum_{(C \rightarrow D E) \in R} \operatorname{Left}(D) \operatorname{Center}(E)+\operatorname{Center}(D) \operatorname{Right}(E)
$$

Let us look at this system as a system of $\mathbb{F}_{2}[[a, b]]$-linear equations over variables Center $(C)=\operatorname{asSeries}\left(L\left(C_{a \rightarrow b}\right)\right)$ for every nonterminal $C$ of the original $\mathrm{GF}(2)$-grammar.

We will consider final $(C)$, $\operatorname{Left}(C)$ and $\operatorname{Right}(C)$ to be the coefficients of the system. While we do not know their exact values, the following is $\operatorname{known}: \operatorname{final}(C)$ is 0 or $b$, $\operatorname{Left}(C) \in \mathcal{A}$ as a formal power series that corresponds to a 2-automatic language over an alphabet $\{a\}$ and, similarly, $\operatorname{Right}(C) \in \mathcal{B}$. That means that all cooeficients of the system lie in $\mathcal{A} \cup \mathcal{B}$ and, therefore, in $R_{a, b}$. The latter is a field by Lemma 3.2.

Denote is the number of nonterminals in the original GF(2)-grammar by $n$, (so there are $n$ nonterminals of type $a \rightarrow b$ in the new GF(2)-grammar), a column vector of values Center $(C)$ by $x$ and a column vector of values final $(C)$ in the same order by $f$. Let us fix the numeration of nonterminals $C$ of the old GF(2)-grammar. After that, we can use them as "numbers" of rows and columns of matrices.

Let $I$ be an identity matrix of dimension $n \times n, A$ be a $n \times n$ matrix with the sum of Left $(D)$ over all rules $C \rightarrow D E$ of the original grammar standing on the intersection of $C$-th row and $E$-th column:

$$
A_{C, E}:=\sum_{(C \rightarrow D E) \in R} \operatorname{Left}(D)
$$

Similarly, let $B$ be a $n \times n$ matrix with

$$
B_{C, D}:=\sum_{(C \rightarrow D E) \in R} \operatorname{Right}(E)
$$

Then, the equation System (2) can be rewritten as $x=f+(A+B) x$ in the matrix form. In other words, $(A+B+I) x=f$. Consider a homomorphism $h: \mathbb{F}_{2}[[a, b]] \rightarrow \mathbb{F}_{2}$ that maps power series to their constant terms (coefficients before $\left.a^{0} b^{0}\right)$. Then, $h(\operatorname{det}(A+B+I))=$ $\operatorname{det}(h(A+B+I))=\operatorname{det}(h(A)+h(B)+h(I))$, where $h$ is extended to the $n \times n$ matrices with components from $\mathbb{F}_{2}[[a, b]]$ in the natural way (replace each component of the matrix by its constant term).

Because the new GF(2)-grammar for $K$ is also in Chomsky normal form, all languages $L\left(C_{a \rightarrow a}\right)$ and $L\left(C_{b \rightarrow b}\right)$ do not contain an empty word. Therefore, all series $\operatorname{Left}(C)=$ $\operatorname{asSeries}\left(L\left(C_{a \rightarrow a}\right)\right)$ and $\operatorname{Right}(C)=\operatorname{asSeries}\left(L\left(C_{b \rightarrow b}\right)\right)$ have zero constant terms. Hence, $h(A)=h(B)=0$, where by 0 we mean a zero $n \times n$ matrix. On the other hand, $h(I)=$ I. Hence, $h(\operatorname{det}(A+B+I))=\operatorname{det}(h(A)+h(B)+h(I))=\operatorname{det}(I)=1$. Therefore, $\operatorname{det}(A+B+I) \neq 0$, because $h(0)=0$. 
Hence, the System (2) has exactly one solution within the field $\mathbb{F}_{2}((a, b))$ - the actual values of Center $(C)$. Moreover, we know that all coefficients of the system lie in the field $R_{a, b} \subset \mathbb{F}_{2}((a, b))$. Therefore, all components of the unique solution also lie within the field $R_{a, b}$. Hence, $\operatorname{asSeries}(K)=\operatorname{asSeries}\left(L\left(S^{\prime}\right)\right)=\operatorname{asSeries}\left(L\left(S_{a \rightarrow a}\right)\right)+\operatorname{asSeries}\left(L\left(S_{a \rightarrow b}\right)\right)$ also lies in $R_{a, b}$.

Remark. Alternatively, one can prove uniqueness of the solution to System (2) by some kind of fixed-point argument. However, I stick to proving that determinant is non-zero, mainly because the proof of Theorem 4.1 still uses Cramer's rule regardless of how the uniqueness of the solution is established.

\section{$3.2 \quad$ Using Theorem 3.1}

It is hard to use Theorem 3.1 directly. Therefore we will prove the following intermediate result:

Theorem 3.2. Suppose that $L \subset a^{*} b^{*}$ is described by a GF(2)-grammar. Denote "the coefficient" of asSeries $(L)$ before $a^{i}$ by $\ell(i) \in \mathbb{F}_{2}[[b]]$, in the sense that $\operatorname{asSeries}(L)=\sum_{i=0}^{+\infty} a^{i} \ell(i)$. Then, there exists a nonnegative integer $d$ and polynomials $p_{0}, p_{1}, \ldots, p_{d} \in \mathbb{F}_{2}[b]$, such that $p_{d} \neq 0$ and $\sum_{i=0}^{d} p_{i} \ell(n-i)$ obtains only finite number of distinct values, when $n$ ranges over the set of all integers larger than $d$.

Example 3.1. For example, suppose that asSeries $(L)=\frac{A_{1} B_{1}+A_{2} B_{2}}{1+a b}$, where $A_{1}, A_{2} \in A$ and $B_{1}, B_{2} \in \mathcal{B}$. Then asSeries $(L)(1+a b)=A_{1} B_{1}+A_{2} B_{2}$. Denote the coefficient of $\operatorname{asSeries}(L)$ befoe $a^{n}$ by $\ell(n)$. Then $\left(\sum_{n=0}^{+\infty} a^{n} \ell(n)\right)(1+a b)=A_{1} B_{1}+A_{2} B_{2}$. Coefficients of the left-hand side before $a^{n}$ are $b \cdot \ell(n-1)+\ell(n)$ for $n \geqslant 1$. Corresponding coefficients of the right-hand side are always from the set $\left\{0, B_{1}, B_{2}, B_{1}+B_{2}\right\}$. Therefore, it is enough to choose $d=1, p_{0}=1, p_{1}=b$ in this case.

Remark. Actually, $\sum_{i=0}^{d} p_{i} \ell(n-i)$ is 2-automatic sequence [1] of elements of $\mathcal{B}$. That is, only elements from $\mathcal{B}$ appear in this sequence, only finite number of them actually appear, and every element appears on 2-automatic set of positions. We just will not need the result in the maximum possible strength here.

Proof of Theorem 3.2. As we already know, $\operatorname{asSeries}(L) \in R_{a, b}$, meaning that $\operatorname{asSeries}(L)=\left(\sum_{k=1}^{K} A_{k} B_{k}\right)\left(\sum_{i=0}^{d} a^{i} p_{i}\right)$ for some nonnegative integers $d, K, A_{i} \in \mathcal{A}, B_{i} \in \mathcal{B}$ and $p_{i} \in \mathbb{F}_{2}[b]$. Moreover, we can choose $d$ in such a way, that $p_{d} \neq 0$ : not all $p_{i}$ are equal to zero, because otherwise the denominator of the fraction would be equal to zero. Also, $\operatorname{asSeries}(L)=\sum_{j=0}^{+\infty} a^{j} \ell(j)$ by definition of $\ell(\cdot)$. 
Therefore, $\left(\sum_{i=0}^{d} a^{i} p_{i}\right) \cdot\left(\sum_{j=0}^{+\infty} a^{j} \ell(j)\right)=\sum_{k=1}^{K} A_{k} B_{k}$. The coefficients of the left-hand and the right-hand sides before $a^{n}$ are $\sum_{i=0}^{\min (n, d)} p_{i} \ell(n-i)$ and $\sum_{a^{n} \in A_{k}} B_{k}$. When $n \geqslant d$, the former of this values is $\sum_{i=0}^{d} p_{i} \ell(n-i)$ and the latter always takes one of $2^{K}$ possible values.

Let us consider a simple application of Theorem 3.2 firstly.

Theorem 3.3. The language $K=\left\{a^{2^{n}} b^{2^{n}} \mid n \in \mathbb{N}\right\}$ is not described by a GF(2)-grammar. Proof. By contradiction. Let us use Theorem 3.2 on the language $K$. The coefficient $\ell(n)$ of $\operatorname{asSeries}(K)$ is $b^{n}$, if $n$ is a power of two and 0 otherwise. In any case, it is divisible by $b^{n}$. From the conclusion of the theorem 3.2, $\sum_{i=0}^{d} p_{i} \ell(n-i)$ obtains only finite number of values for some integer $d$ and $p_{0}, p_{1}, \ldots, p_{d} \in \mathbb{F}_{2}[b]$, satisfying the condition $p_{d} \neq 0$.

On the other hand, the sum $\sum_{i=0}^{d} p_{i} \ell(n-i)$ is divisible by $b^{n-d}$ : every summand contains a multiplier $\ell(n-i)$, which is divisible by $b^{n-i}$. Therefore, $\sum_{i=0}^{d} p_{i} \ell(n-i)$ is divisible by larger and larger powers of $b$ as $n$ grows. Therefore, the only value that this sum can obtain infinitely often is 0 : other power series are not divisible by arbitrarily large powers of $b$. Because the sum obtains only finite number of values, 0 is obtained for large enough $n$.

Therefore, some fixed linear combination of $\ell(n-d), \ell(n-d+1), \ldots, \ell(n)$ is equal to 0 for large enough $n$. However, in the sequence $\ell(i)$ appear very rarely: the gaps between them grow larger and larger. In particular, one can choose such $n \geqslant d$, that $\sum_{i=0}^{d} p_{i} \ell(n-i)=0$, $\ell(n-d) \neq 0$, but $\ell(n-d+1)=\ldots=\ell(n)=0$. This is impossible, because $p_{d} \neq 0$ and therefore there is exactly one non-zero summand in zero sum: $p_{d} \ell(n-d)$.

To be exact, one can pick $n=d+2^{m}$ for large enough $m$.

A more interesting application of the technique can be seen here:

Theorem 3.4. Suppose that $f: \mathbb{N} \rightarrow \mathbb{N}$ is a strictly increasing function. If a language $L_{f}=\left\{a^{n} b^{f(n)} \mid n \in \mathbb{N}\right\}$ is generated by a GF(2)-grammar, then the set $f(\mathbb{N})$ is a finite union of arithhmetical progressions.

Proof. Let us use the Theorem 3.2. The proof is structured in the following way. The first step is to prove that polynomials $b^{f(n)}$ satisfy some linear reccurence that has rational functions of $b$ as coefficients. The second step is to prove that in this case $f(\mathbb{N})$ is indeed a finite union of arithmetical progressions. Intuitively, it is hard to imagine a linear recurrence with all values looking like $b^{\text {something }}$, but without strong regularity properties. 
Let us use Theorem 3.2 on the language $L_{f}$. The coefficient of asSeries $\left(L_{f}\right)$ before $a^{n}$ is $b^{f(n)}$. Therefore, $\sum_{i=0}^{d} p_{i} b^{f(n-i)}$ obtains only finite number of values for some nonnegative integer $d$ and some $p_{0}, p_{1}, \ldots, p_{d} \in \mathbb{F}_{2}[b]$ satisfying the property $p_{d} \neq 0$. Notice that this sum starts being divisible by arbitrarily large powers of $b$ when $n$ increases (here we use the fact that $f$ is an increasing function). Because the sum obtains only finite number of values, it is equal to zero for large enough $n$.

Now, we want to prove that $\{f(n) \mid n \in \mathbb{N}\}$ is a finite union of arithmetical progressions. We already established that $\sum_{i=0}^{d} p_{i} b^{f(n-i)}=0$ for large enough $n$.

Let $j$ be the smallest index, such that $p_{j} \neq 0$ : it exists, because $p_{d} \neq 0$. Moreover, $j \neq d$, otherwise $0=p_{d} b^{f(n-d)}$ for large enough $n$, contradicting $p_{d} \neq 0$. Therefore, $p_{j} b^{f(n-j)}=\sum_{i=j+1}^{d} p_{i} b^{f(n-i)}$. Let us rewrite the last statement in a slightly different way: $b^{f(n-j)}=\sum_{i=j+1}^{d} \frac{p_{i}}{p_{j}} b^{f(n-i)}$.

Divide both sides of the last equality by $b^{f(n-d)}$. Then, $b^{f(n-j)-f(n-d)}=$ $\sum_{i=j+1}^{d} \frac{p_{i}}{p_{j}} b^{f(n-i)-f(n-d)}$. Therefore, the difference $f(n-j)-f(n-d)$ depends only on differences $f(n-j-1)-f(n-d), \ldots, f(n-d+1)-f(n-d)$, but not on $f(n-d)$ itself.

Now, let us prove that the difference $f(n+1)-f(n)$ is bounded above (it is always positive, because $f$ is increasing). Indeed, as we know, $\sum_{i=0}^{d} p_{i} b^{f(m-i)}=0$ for large enough $m$. Substitute $n=m-d$ and $k=d-i$, the result is $\sum_{k=0}^{d} p_{d-k} b^{f(n+k)}=0$. Because $f$ increases, all summands are divisible by $b^{f(n+1)}$, with possible exception of the first summand. Because the sum is equal to 0 , the first summand should be divisible by $b^{f(n+1)}$ as well. Therefore, it is not equal to 0 (because $p_{d} \neq 0$ and $b^{f(n)} \neq 0$ ) and its degree as a polynomial of $b$ is equal to $\operatorname{deg} p_{d}+\operatorname{deg} b^{f(n)}=\operatorname{deg} p_{d}+f(n)$. The degree of a non-zero polynomial divisible by $b^{f(n+1)}$ is at least $f(n+1)$, therefore $f(n+1)-f(n) \leqslant \operatorname{deg} p_{d}$.

Because differences $f(n+1)-f(n)$ are bounded, then differences $f(n+k)-f(n)=$ $(f(n+k)-f(n+k-1))+(f(n+k-1)-f(n+k-2))+\ldots+(f(n+1)-f(n))$ are bounded as well for all $k \leqslant d$. Therefore, the tuple of differences $(f(n-j-1)-f(n-$ $d), \ldots, f(n-d+1)-f(n-d))$ obtains only finite set of values as $n$ goes towards infinity. As shown above, $f(n-j)-f(n-d)$ is uniquely restorable from such a tuple. Therefore, this tuple for $n+1$ is uniquely restorable from this tuple for $n$ : we need to know only some pairwise differences between elements of $\{f(n-j), f(n-j-1), \ldots, f(n-d)\}$ and all such differences are determined by differences, where the smaller number is $f(n-d)$.

Because there is only finite number of such tuples, and each tuple determines the next, they start "going in circles" at some moment. In particular, differences $f(n-d+1)-f(n-d)$ start going in circles. This, along with $f$ being increasing, is enough to establish that $\{f(n) \mid n \in \mathbb{N}\}$ is a finite union of arithmetic progressions. 


\section{Subsets of $a^{*} b^{*} c^{*}$}

The language $\left\{a^{n} b^{n} c^{n} \mid n \geqslant 0\right\}$ is, probably, the most famous example of a simple language that is not described by any ordinary grammar. It is reasonable to assume that it is not described by a $\mathrm{GF}(2)$-grammar as well. Let us prove that.

We will do more than that and will actually establish some property that all subsets of $a^{*} b^{*} c^{*}$ that can be described by a GF(2)-grammar have, but $\left\{a^{n} b^{n} c^{n} \mid n \geqslant 0\right\}$ does not. Most steps of the proof will be analogous to the two-letter case.

There is a natural one-to-one correspondence between subsets of $a^{*} b^{*} c^{*}$ and formal power series in variables $a, b$ and $c$ over field $\mathbb{F}_{2}$. Indeed, for every set $S \subset \mathbb{N}_{0}^{3}$, we can identify the language $\left\{a^{n} b^{m} c^{k} \mid(n, m, k) \in S\right\} \subset a^{*} b^{*} c^{*}$ with the formal power series

$\sum_{(n, m, k) \in S} a^{n} b^{m} c^{k}$. Denote this correspondence by asSeries: $2^{a^{*} b^{*} c^{*}} \rightarrow \mathbb{F}_{2}[[a, b, c]]$. Then, $\operatorname{asSeries}(L \triangle K)=\operatorname{asSeries}(L)+\operatorname{asSeries}(K)$. In other words, the symmetric difference of languages corresponds to the sum of formal power series.

Similarly to the Lemma 3.1, $\operatorname{asSeries}(K \odot L)=\operatorname{asSeries}(K) \cdot \operatorname{asSeries}(L)$ in the following important special cases: when $K$ is a subset of $a^{*}$, when $K$ is a subset of $a^{*} b^{*}$ and $L$ is a subset of $b^{*} c^{*}$, and, finally, when $L$ is a subset of $c^{*}$. Indeed, in each of these three cases, symbols "are in the correct order": if $u \in K$ and $v \in L$, then $u v \in a^{*} b^{*} c^{*}$.

However, we cannot insert character $b$ in the middle of the string: if $K$ is a subset of $b^{*}$ and $L$ is a subset of $a^{*} b^{*} c^{*}$, then $K \odot L$ does not even have to be a subset of $a^{*} b^{*} c^{*}$.

The "work plan" will remain the same as in the previous section: we will prove that some algebraic structure is a field and then use linear algebra over said field. Let us get two possible questions out of the way first:

1. Why is it logical to expect that the language $\left\{a^{n} b^{n} c^{n} \mid n \geqslant 0\right\}$ is not described by a $\mathrm{GF}(2)$-grammar, but a similar language $\left\{a^{n} b^{n} \mid n \geqslant 0\right\}$ is?

2. Why will the proof work out for $\left\{a^{n} b^{n} c^{n} \mid n \geqslant 0\right\}$, but not for a regular language $\left\{(a b c)^{n} \mid n \geqslant 0\right\}$, despite these languages having the same "commutative image"?

1. Simply speaking, the reason is the same as for the ordinary grammars. On intuitive level, both ordinary grammars and $\mathrm{GF}(2)$-grammars permit a natural way to "capture" the events that happen with any two letters in subsets of $a^{*} b^{*} c^{*}$, but not all three letters at the same time. A rigourous result that corresponds to this intuitive limitation of ordinary grammars was proven by Ginsburg and Spanier [10, Theorem 2.1]. Theorem 4.1 is an analogue for $\mathrm{GF}(2)$-grammars.

2. This argument only implies that any proof that relies solely on commutative images is going to fail. The real proof is more subtle. For example, it will also use the fact that $\left\{a^{n} b^{n} c^{n} \mid n \geqslant 0\right\}$ is a subset of $a^{*} b^{*} c^{*}$.

While the proof uses commutative images, it uses them very carefully, always making sure that the letters "appear in the correct order". In particular, we will never consider 
GF(2)-concatenations $K \odot L$, where $K$ is a subset of $b^{*}$ and $L$ is an arbitrary subset of $a^{*} b^{*} c^{*}$, in the proof, because in this case $K \odot L$ is not a subset of $a^{*} b^{*} c^{*}$.

Avoiding this situation is impossible for language $\left\{(a b c)^{n} \mid n \geqslant 0\right\}$, because in the word $a b c a b c$ from this language the letters "appear in the wrong order".

Denote the set of algebraic power series in variable $c$ by $\mathcal{C}$, the set of polynomials in variables $a$ and $c$ by $\operatorname{poly}(a, c)$, et cetera.

Similarly to Definition 3, define $R_{a, c} \subset \mathbb{F}_{2}((a, c))$ and $R_{b, c} \subset \mathbb{F}_{2}((b, c))$.

Finally, denote by $R_{a, b, c}$ the set of all Laurent series that can be represented as $\frac{\sum_{i=1}^{n} A_{i} B_{i} C_{i}}{p_{a, b} \cdot p_{a, c} \cdot p_{b, c}}$, where $n$ is a nonnegative integer, $A_{i} \in \mathcal{A}, B_{i} \in \mathcal{B}, C_{i} \in \mathcal{C}$ for all $i$ from 1 to $n$, and $p_{a, b} \in \operatorname{poly}(a, b), p_{a, c} \in \operatorname{poly}(a, c), p_{b, c} \in \operatorname{poly}(b, c)$.

Lemma 4.1. $R_{a, b, c}$ is a subring of $\mathbb{F}_{2}((a, b, c))$. Moreover, $R_{a, b}, R_{a, c}$ and $R_{b, c}$ are subsets of $R_{a, b, c}$.

Sketch of a proof. It is easy to see that $R_{a, b, c}$ is closed under addition and multiplication. Setting $C_{1}=C_{2}=\ldots=C_{n}=p_{a, c}=p_{b, c}=1$ yields $R_{a, b} \subset R_{a, b, c}$.

\subsection{Main result}

Unlike $R_{a, b}, R_{a, b, c}$ is not a field (in fact, Subsection 4.2 tells us that $(1+a b c)^{-1} \notin R_{a, b, c}$ ), so a bit more involved argument will be necessary for the proof of the following theorem:

Theorem 4.1. Suppose that $K \subset a^{*} b^{*} c^{*}$ is described by a GF(2)-grammar. Then the corresponding formal power series $\operatorname{asSeries}(K)$ is in the set $R_{a, b, c}$.

Proof outline. The proof is mostly the same as the proof of Theorem 3.1. Let us focus on the differences. As before, we can assume that $K$ does not contain an empty word.

In the same manner, we formally intersect our GF(2)-grammar in Chomsky's normal form with the language $a^{*} b^{*} c^{*}$. Now, all nonterminals $C$ of the original GF(2)-grammar split into six nonterminals: $C_{a \rightarrow a}, C_{a \rightarrow b}, C_{a \rightarrow c}, C_{b \rightarrow b}, C_{b \rightarrow c}, C_{c \rightarrow c}$. However, their meanings stay the same: for example, $L\left(C_{a \rightarrow b}\right)=L(C) \cap\left(a^{*} b^{+}\right)$and $L\left(C_{a \rightarrow c}\right)=L(C) \cap\left(a^{*} b^{*} c^{+}\right)$.

However, only the "central" nonterminals $C_{a \rightarrow c}$ are important, similarly to the nonterminals of the type $a \rightarrow b$ in the proof of Theorem 3.1. Why? Before, we had some a priori knowledge about the languages $L\left(C_{a \rightarrow a}\right)$ and $L\left(C_{b \rightarrow b}\right)$ from Christol's theorem. But now, because of Theorem 3.1, we have a priori knowledge about the languages $L\left(C_{a \rightarrow b}\right)$ and $L\left(C_{b \rightarrow c}\right)$ as well, because they are subsets of $a^{*} b^{*}$ and $b^{*} c^{*}$ respectively.

Remark. In a sense, we used Theorem $C$ as a stepping stone towards the proof of Theorem 3.1, and now we can use Theorem 3.1 as a stepping stone towards the proof of Theorem 4.1. 
Denote by end $(C)$ the language $\left(\bigoplus_{(C \rightarrow D E) \in R} L\left(D_{a, b}\right) \odot L\left(E_{b, c}\right)\right) \oplus T_{C}$, where $T_{C}$ is either $\{c\}$ or $\varnothing$, depending on whether or not there is a "final" rule $C_{a \rightarrow c} \rightarrow c$ in the new $\mathrm{GF}(2)$ grammar.

This means that we again can express the values $\operatorname{asSeries}\left(L\left(C_{a \rightarrow c}\right)\right)$ as a solution to a system of linear equations with relatively simple coefficients (denote asSeries $\left(L\left(C_{a \rightarrow c}\right)\right.$ ) by $\operatorname{Center}(C)$, asSeries $\left(L\left(C_{a \rightarrow a}\right)\right)$ by $\operatorname{Left}(C)$, $\operatorname{asSeries}\left(L\left(C_{c \rightarrow c}\right)\right)$ by $\operatorname{Right}(C)$ and asSeries $(\operatorname{end}(C))$ by final $(C))$ :

$$
\operatorname{Center}(C)=\operatorname{final}(C)+\sum_{(C \rightarrow D E) \in R} \operatorname{Left}(D) \operatorname{Center}(E)+\operatorname{Center}(D) \operatorname{Right}(E)
$$

Here, the summation is over all rules $C \rightarrow D E$ of the original GF(2)-grammar. Similarly to the proof of Theorem 3.1, this system can be rewritten as $(A+B+I) x=f$, where $x$ and $f$ are column-vectors of $\operatorname{Center}(C)$ and final $(C)$ respectively, while the matrices $A$ and $B$ are defined as follows:

$$
\begin{aligned}
A_{C, E} & :=\sum_{C \rightarrow D E} \operatorname{Left}(D) \\
B_{C, E} & :=\sum_{C \rightarrow D E} \operatorname{Right}(E)
\end{aligned}
$$

Again, this system has a unique solution, because we can prove that $\operatorname{det}(A+B+I) \neq 0$ in the same way as before. Because $\operatorname{det}(A+B+I) \neq 0$, then, by Cramer's formula, each component of the solution, Center $(S)$, in particular, can be represented in the following form:

$$
\frac{\operatorname{det}(A+B+I, \text { but one of the columns was replaced by } f)}{\operatorname{det}(A+B+I)}
$$

The numerator of the above fraction is the determinant of some matrix, all whose components are lie the ring $R_{a, b, c}$ by Lemma 4.1. The denominator, on the other hand lies in the field $R_{a, c}$, because all components of $A+B+I$ lie in $\mathcal{A} \cup \mathcal{C} \subset R_{a, c}$. Because $R_{a, c}$ is a field, the inverse of the denominator also lies in $R_{a, c}$. Hence, $\operatorname{Center}(S)$ is a product of an element of $R_{a, b, c}$ and an element of $R_{a, c}$. Hence, Center $(S) \in R_{a, b, c}$. Therefore, $\operatorname{asSeries}(K)=\operatorname{asSeries}\left(L\left(S^{\prime}\right)\right)=\operatorname{asSeries}\left(L\left(S_{a \rightarrow a}\right)\right)+\operatorname{asSeries}\left(L\left(S_{a \rightarrow b}\right)\right)+\operatorname{Center}(S)$ also lies in the set.

Consider the case of larger alphabets. Let $\mathcal{A}_{i}$ be the set of all algebraic formal power series in variable $a_{i}$. Similarly to $R_{a, b, c}$, denote by $R_{a_{1}, a_{2}, \ldots, a_{k}}$ the set of all Laurent series that can be represented as $\frac{\sum_{i=1}^{n} A_{i, 1} A_{i, 2} \ldots A_{i, k}}{\prod_{1 \leqslant i<j \leqslant n} p_{i, j}}$, for some $n \geqslant 0, p_{i, j} \in \operatorname{poly}\left(a_{i}, a_{j}\right)$ and $A_{i, j} \in \mathcal{A}_{j}$

Theorem 4.2. If a language $K \subset a_{1}^{*} a_{2}^{*} \ldots a_{k}^{*}$ is described by a GF(2)-grammar, then the corresponding power series $\operatorname{asSeries}(K)$ is in the set $R_{a_{1}, a_{2}, \ldots, a_{k}}$. 
Sketch of the proof. Induction over $k$, the induction step is analogous to the way we used Theorem 3.1 in the proof of Theorem 4.1.

\subsection{The language $\left\{a^{n} b^{n} c^{n} \mid n \geqslant 0\right\}$ and its relatives}

In this subsection, we will use our recently obtained knowledge to prove that there is no $\mathrm{GF}(2)$-grammar for the language $\left\{a^{n} b^{n} c^{n} \mid n \geqslant 0\right\}$. It will almost immediately follow that the languages $\left\{a^{n} b^{m} c^{\ell} \mid n=m\right.$ or $\left.m=\ell\right\}$ and $\left\{a^{n} b^{m} c^{\ell} \mid n \neq m\right.$ or $\left.m \neq \ell\right\}$

Consider the formal power series asSeries $\left(\left\{a^{n} b^{n} c^{n} \mid n \geqslant 0\right\}\right)=\sum_{n=0}^{+\infty} a^{n} b^{n} c^{n}$. Denote these series by $f$ for brevity. It is not hard to see that $f=(1+a b c)^{-1}$. Indeed, $f \cdot(1+a b c)=$ $\sum_{n=0}^{+\infty}\left(a^{n} b^{n} c^{n}+a^{n+1} b^{n+1} c^{n+1}\right)=1$, because all summands except $a^{0} b^{0} c^{0}=1$ cancel out.

It sounds intuitive that $(1+a b c)^{-1}$ "depends" on $a, b$ and $c$ in a way that the expression $\frac{\sum \mathcal{A B C}}{\operatorname{poly}(a, b) \text { poly }(a, c) \text { poly }(b, c)}$ cannot capture; every series that match this expression should "split" nicely into functions that depend only on two of three variables. Now, let us establish that $f$ does not match this expression formally.

Indeed, suppose that it is not true. In other words,

$$
f=\frac{\sum_{i=1}^{n} A_{i} B_{i} C_{i}}{p q r},
$$

where $A_{i} \in \mathcal{A}, B_{i} \in \mathcal{B}, C_{i} \in \mathcal{C}$ for every $i$ from 1 to $n$ and, also, $p \in \operatorname{poly}(a, b), q \in \operatorname{poly}(a, c)$ and $r \in \operatorname{poly}(b, c)$. Let us rewrite the equation 6 as $\operatorname{pqr} f=\sum_{i=1}^{n} A_{i} B_{i} C_{i}$ with an additional condition that neither of $p, q$ and $r$ is zero: otherwise the denominator of the right-hand side of the equation 6 is zero.

For every formal power series of three variables $a, b$ and $c$ we can define its trace: such subset of $\mathbb{N}_{0}^{3}$, that triple $(x, y, z)$ is in this subset if and only if coefficient of the series before $a^{x} b^{y} c^{z}$ is one. Traces of equal power series coincide.

How do the traces of left-hand and right-hand sides of equation $p q r f=\sum_{i=1}^{n} A_{i} B_{i} C_{i}$ look like? Intuitively, the trace of the left-hand side should be near the diagonal $x=y=z$ in its entirety, because $p q r f$ is a polynomial $p q r$, multiplied by $f=\sum_{i=0}^{+\infty} a^{i} b^{i} c^{i}$. On the other hand, the trac e of the right-hand side has "block structure": as we will establish later, it should be a finite union of disjoint sets with type $X \times Y \times Z$.

Our goal is to prove that such traces can coincide only if they are both finite. This conclusion is quite natural: the trace of the left-hand side exhibits "high dependency" between $x, y$ and $z$, while coordinates "are almost independent" in the trace of the righthand side (and they would be "fully independent" if there was only one set $X \times Y \times Z$ in the disjoint union).

Let us proceed formally. 
Lemma 4.2. The trace of the expression $\sum_{i=1}^{n} A_{i} B_{i} C_{i}$ is a finite disjoint union of sets with type $X \times Y \times Z$.

Proof. For $x \in \mathbb{N}_{0}$, let us call the set of all such $i$ from 1 to $n$, that the coefficient of $A_{i}$ before $a^{x}$ is one the $a$-type of $x$. Similarly, define $b$-type and $c$-type.

Whether or not the triple $(x, y, z)$ is in the trace of $\sum_{i=1}^{n} A_{i} B_{i} C_{i}$ depends only on the a-type of $x$, b-type of $y$ and c-type of $z$. Indeed, the coefficient before $a^{x} b^{y} c^{z}$ is one in exactly such summands $A_{i} B_{i} C_{i}$, that the coefficient of $A_{i}$ before $a^{x}$ is one, the coefficient of $B_{i}$ before $b^{y}$ is one and the coefficient of $C_{i}$ before $c^{z}$ is one. Therefore the exact set of such summands depends only on types of $x, y$ and $z$, let alone the parity of their number.

Consequently, the trace of $\sum_{i=1}^{n} A_{i} B_{i} C_{i}$ is a union of sets $X \times Y \times Z$, where $X$ is a set of numbers with some fixed $a$-type, $Y$ is a set of numbers with some fixed $b$-type and $Z$ is a set of numbers with some fixed $c$-type. There is only finite number of such sets, because there is no more than $2^{n}$ a-types, no more than $2^{n}$ b-types and no more than $c$-types.

Lemma 4.3. There exists a such constant $d$, that for every triple $(x, y, z)$ from trace of pqrf conditions $|x-y| \leqslant d,|x-z| \leqslant d$ and $|y-z| \leqslant d$ hold.

Proof. Let $d$ be the degree of $p q r$ as of a polynomial of three variables. Because pqr $f=$ $p q r \cdot \sum_{i=0}^{+\infty} a^{i} b^{i} c^{i}$, the trace of pqrf may only contain triples $(\ell+i, m+i, k+i)$ for monomials $a^{\ell} b^{m} c^{k}$ from the polynomial pqr. For such triples, $|x-y|=|\ell-m| \leqslant d$, because $d$ is degree of $p q r$ and, therefore, $0 \leqslant \ell \leqslant d$ and $0 \leqslant m \leqslant d$. Similarly, $|x-z| \leqslant d$ and $|y-z| \leqslant d$.

Lemma 4.4. If traces of $\sum_{i=1}^{n} A_{i} B_{i} C_{i}$ and pqrf coincide, then they both are finite sets.

Proof. From Lemmata 4.2 and 4.3, a set that is close to the diagonal coincides with a disjoint union of sets of a type $X \times Y \times Z$. Then, each of the sets $X \times Y \times Z$ in the union is finite. Roughly speaking, infinite sets of such type should contain elements that are arbitrarily far from the diagonal $x=y=z$.

Let us explain the previous paragraph more fomally. Indeed, suppose that some of the $X \times Y \times Z$ set is infinite. Then, at least one of the sets $X, Y$ and $Z$ is infinite. Without loss of generality, $X$ is infinite. Let $(x, y, z)$ be some element of $X \times Y \times Z$ : it exists, because every infinite set contains at least one element. Choose $x_{\text {new }}$ so $x_{\text {new }}>\max (y, z)+d$. Such $x_{\text {new }}$ exists, because $X$ is infinite set of nonnegative integers. Then, $\left(x_{\text {new }}, y, z\right) \in X \times Y \times Z$. Therefore, $\left(x_{\text {new }}, y, z\right)$ is in the trace of $\sum_{i=1}^{n} A_{i} B_{i} C_{i}$. However, by Lemma $4.3,\left(x_{\mathrm{new}}, y, z\right)$ cannot lie in the trace of $p q r f$, because $x_{\text {new }}$ differs from $y$ and $z$ too much.

Lemma 4.5. The polynomial $1+$ abc is irreducible as a polynomial over field $\mathbb{F}_{2}$.

Proof. Suppose that $1+a b c$ is irreducible. Because its total degree is 3 , it should split into a product of two polynomials with total degrees 1 and 2 respectively. In principle, 
enumerating all pairs of polynomials over $\mathbb{F}_{2}$ of total degree 1 and 2 on the computer does the job. One may be interested in a proof without computer search, though.

Because the degree of $1+a b c$ with respect to each variable is 1 , each variable occurs in exactly one of two factors - if it occurs in both, the resulting degree is at least 2, if it occurs in neither, the resulting degree is 0 .

Because the total degree of the second factor is 2 , but its degree in every variable is only 1 , exactly 2 variables occur in the second factor. Therefore, only one variable occurs in the first factor. Because the polynomial $1+a b c$ is symmetric with respect to permutation in variables, we may assume that the first factor depends only on $a$ and the second factor depends only on $b$ and $c$.

The first factor is invertible, because the product is invertible. Previously, we have shown that the first factor is a polynomial of $a$ of degree 1, therefore the only one possibility for the first factor remains: $1+a$. The second factor is also invertible and is of degree 2 , therefore it is $1+k_{b} b+k_{c} c+b c$ for some $k_{b}$ and $k_{c}$ from $\mathbb{F}_{2}$. Then, there is a summand $a \cdot 1=a$ in their product, which does not have anything to cancel up with. But their product is $1+a b c$, contradiction.

Because $p q r f=\sum_{i=1}^{n} A_{i} B_{i} C_{i}$, the trace of pqrf is finite. In other words, pqrf is a polynomial. Recall that $f=(1+a b c)^{-1}$, so $\frac{p q r}{1+a b c}$ is a polynomial. Because the product of three polynomials $p, q$ and $r$ is divisible by an irreducible polynomial $1+a b c$, one of them is also divisible by $1+a b c$. But this is impossible, because each of polynomials $p, q$ and $r$ is non-zero (here we used that condition, at last) and does not depend on one of the variables.

Finally, we have established the following theorem.

Theorem 4.3. The language $\left\{a^{n} b^{n} c^{n} \mid n \geqslant 0\right\}$ is not described by a GF(2)-grammar.

Corollary. The language $\left\{a^{n} b^{m} c^{\ell} \mid n=m\right.$ or $\left.m=\ell\right\}$ is not described by a GF(2)grammar.

Proof. Suppose that $\left\{a^{n} b^{m} c^{\ell} \mid n=m\right.$ or $\left.m=\ell\right\}$ is described by a GF(2)-grammar. Then, $\left\{a^{n} b^{n} c^{n} \mid n \geqslant 0\right\}$ also is, as symmetric difference of $\left\{a^{n} b^{m} c^{\ell} \mid n=m\right.$ or $\left.m=\ell\right\}$ and $\left\{a^{n} b^{m} c^{\ell} \mid n=m\right.$ or $m=\ell$, but not both $\}$, where the latter is described by a GF(2)grammar [15, Example 2]. Contradiction.

Corollary. The language $\left\{a^{n} b^{m} c^{\ell} \mid n \neq m\right.$ or $\left.m \neq \ell\right\}$ is not described by a GF(2)grammar.

Proof. Otherwise, $\left\{a^{n} b^{n} c^{n} \mid n \geqslant 0\right\}=\left(a^{*} b^{*} c^{*}\right) \triangle\left\{a^{n} b^{m} c^{\ell} \mid n \neq m\right.$ or $\left.m \neq \ell\right\}$ would be described by a $\mathrm{GF}(2)$-grammar as well.

We have just proven that the language $\left\{a^{n} b^{m} c^{\ell} \mid n=m\right.$ or $\left.m=\ell\right\}$ is not described by a GF(2)-grammar. Hence, it is inherently ambiguous. Previous proofs of its inherent ambiguity were purely combinatorial, mainly based on Ogden's lemma, while our approach is mostly algebraic. 
What is even more important, we proved that the language $\left\{a^{n} b^{m} c^{\ell} \mid n \neq m\right.$ or $\left.m \neq \ell\right\}$ is not described by a GF(2)-grammar, therefore inherently ambiguous. Inherent ambiguity of that language was not known before.

\section{$5 \quad$ Are converse statements true?}

It would be interesting to know whether the converse statements to Theorems 3.1, 4.1 and 4.2 are true. Of course, the exact converse statement to Theorem 3.1 is wrong on the technicality that not all elements of $R_{a, b}$ are power series. However, if we restrict ourselves to $R_{a, b} \cap \mathbb{F}_{2}[[a, b]]$, we get the following conjecture, which I believe in:

Conjecture 1. If $f \in R_{a, b} \cap \mathbb{F}_{2}[[a, b]]$, then the language asSeries ${ }^{-1}(f) \subset a^{*} b^{*}$ can be described by a $G F(\mathscr{Q})$-grammar.

The following Theorem 5.1 is an evidence in favor of Conjecture 1 . We will need the following definition to state Theorem 5.1.

Definition. Denote by $R_{a, b}^{\text {int }}$ the set of Laurent series from $\mathbb{F}_{2}((a, b))$ that can be represented as $\frac{\sum_{i=1}^{n} A_{i} B_{i}}{p}$, where $n \geqslant 0, A_{i} \in \mathcal{A}$ and $B_{i} \in \mathcal{B}$ for $i$ in range from 1 to $n$, and $p \in \operatorname{poly}(a, b)$ is a polynomial with constant term equal to 1.

Remark. The only difference between the definitions of $R_{a, b}$ and $R_{a, b}^{\text {int }}$ is that the denominator $p$ of the fraction is required to be invertible as an element of $\mathbb{F}_{2}[[a, b]] \supset$ poly $(a, b)$. In particular, $R_{a, b}^{\text {int }}$ is a subset of $\mathbb{F}_{2}[[a, b]]$. Also, by definition, $R_{a, b}^{\text {int }} \subset R_{a, b}$.

Theorem 5.1. If $f \in R_{a, b}^{\text {int }}$, then asSeries $^{-1}(f)$ can be described by a GF(2)-grammar.

Sketch of the proof. Suppose that $f=\left(\sum_{i=1}^{n} A_{i} B_{i}\right) / p$, as in Definition 5 , and $p=1+$ $\sum_{j=1}^{d} a^{k_{j}} b^{\ell_{j}}$, where $k_{j}+\ell_{j}>0$. Let $S$ be the starting symbol of some GF(2)-grammar that describes asSeries $^{-1}\left(\sum_{i=1}^{n} A_{i} B_{i}\right)$ (such GF (2)-grammar exists by closure properties). Add a new starting symbol $S_{\text {new }}$ and a new rule $S_{\text {new }} \rightarrow\left(\oplus_{j=1}^{d} a^{k_{j}} S_{\text {new }} b^{\ell_{j}}\right) \oplus S$ to the $\mathrm{GF}(2)$ grammar. The new GF(2)-grammar describes asSeries ${ }^{-1}(f)$. The condition $k_{j}+\ell_{j}>0$ is important, because it assures that all words still have only a finite number of parse trees.

Hence, the only thing that stops us from proving Conjecture 1 is the following conjecture, which I, personally, believe in:

Conjecture 2. $R_{a, b}^{i n t}=R_{a, b} \cap F[[a, b]]$.

This is a purely algebraic statement. Moreover, it seems like either false statement, or one that should easily follow from well-known algebra. However, I could neither find an independent proof of Conjecture 2, nor derive it from some more general algebra, nor disprove it. Maybe the readers will be more successful?

On the contrary, I do not believe in analogous statements for three and more letters. For four and more letters there is a simple potential counterexample. 
Conjecture 3. $f:=\left(\sum_{n=0}^{+\infty} \sum_{m=0}^{+\infty} a^{n} b^{m} c^{n} d^{m}\right)=\frac{1}{(1+a c)(1+b d)}$ is in the set $R_{a, b, c, d}$, but asSeries $^{-1}(f)$ cannot be described by a $G F($ 2)-grammar.

Why is it a potential counterexample? It is hard to imagine GF(2)-grammars actually handling "overlapping requirements" about letters $a, c$ and $b, d$. It is well-known that ordinary grammars cannot do that [10].

The case of three letters is less clear, but I still believe that there is such a language.

Conjecture 4. There exists $f \in R_{a, b, c} \cap \mathbb{F}_{2}[[a, b, c]]$, such that there is no GF(2)-grammar for $\operatorname{asSeries}^{-1}(f)$.

My thoughts. Three-letter alphabet is some kind of in-between case. The "gut feeling" that the proof of Theorem 4.1 "loses too much information" about the original language is already here, but it is hard to find any counterexample, even a hypothetical one.

\section{Conclusion}

Let us make some concluding remarks and discuss some possible future developments.

Firstly, note that it took us roughly the same effort to prove the inherent ambiguity of $\left\{a^{n} b^{m} c^{\ell} \mid n=m\right.$ or $\left.m=\ell\right\}$ and $\left\{a^{n} b^{m} c^{\ell} \mid n \neq m\right.$ or $\left.m \neq \ell\right\}$, despite the former being a textbook example of inherently ambiguous language and the latter not being known to be inherently ambiguous before. Intuitively, it is very difficult to capture weak conditions like inequality using Ogden's lemma, while our approach can replace inequality with a strong condition (equality) by taking the complement.

Secondly, It would be possible to modify the argument in Subsection 4.2 of this paper to use only Ginsburg's and Ullian's result instead of Theorem 4.1. However, such a proof would not be much simpler, and I do not know how to discover it without using algebraic intuition that comes from the proof that uses GF(2)-grammars.

Thirdly, the proofs of Theorems 3.1 and 4.1 start similarly to the reasoning Ginsburg and Spanier used to characterize bounded languages described by ordinary grammars [10], but diverge after taking some steps. This is not surprising; ordinary grammars have good monotonicity properties (a word needs only one parse tree to be in the language), but bad algebraic properties (solving systems of language equations is much harder than solving systems of linear equations). In GF(2)-grammars, it is the other way around: there are no good monotonicity properties, but algebraic properties are quite remarkable.

Perhaps, our methods could be used to make some progress on the equivalence problem for unambiguous grammars. Indeed, the equivalence problem for unambiguous grammars is closely related to the emptiness problem for $\mathrm{GF}(2)$-grammars. If it is decidable, whether $\mathrm{GF}(2)$-grammar describes an empty language or not, then the equivalence of unambiguous grammars is decidable as well. If it is not, the proof will most probably shed some light on the case of unambiguous grammars anyway. However, resolving the emptiness problem for GF(2)-grammars in one way or another still seems to be out of reach. 
Understanding, how are our methods related to the analytic methods of Flajolet [6], is another interesting question. One can see Theorem $\mathrm{C}$ as an alternative formulation of Christol's theorem [7] for $\mathbb{F}_{2}$ specifically, that involves $\mathrm{GF}(2)$-grammars instead of 2automatic sequences on the "combinatorial side". Then, Christol's theorem can be seen as a finite field analogue of Chomsky-Schützenberger enumeration theorem, because both relate counting properties of different grammar families to algebraic power series over fields by "remembering" only the length of the word, but nothing else:

Theorem $\mathbf{D}$ (Chomsky-Schützenberger enumeration theorem [5]). If $L$ is a language described by an unambiguous grammar, and $a_{k}$ is the number of words of length $k$ in $L$, then the power series $\sum_{k=0}^{+\infty} a_{k} x^{k}$ is algebraic over $\mathbb{Q}[x]$.

Theorem $\mathbf{E}$ (Christol's theorem for $\mathbb{F}_{2}[7,15]$; usually not stated this way). If $L$ is a language described by a GF(2)-grammar, and $a_{k}$ is the number of words of length $k$ in $L$, then the power series $\sum_{k=0}^{+\infty}\left(a_{k} \bmod 2\right) \cdot x^{k}$ is algebraic over $\mathbb{F}_{2}[x]$.

This gives us some hope that our methods can be at least partially transfered to the analytic setting. Moreover, a lot (though not all) of the arguments used in our work can be modified to work over an arbitrary field.

\section{References}

[1] J.-P. Allouche, J. Shallit, Automatic Sequences: Theory, Applications, Generalizations, Cambridge University Press, 2003.

[2] J.-M. Autebert, J. Beauquier, L. Boasson, M. Nivat, "Quelques problèmes ouverts en théorie des langages algébriques", RAIRO - Theoretical Informatics and Applications - Informatique Théorique et Applications, Volume 13, number 4 (1979), 363-378.

[3] E. Bakinova, A. Basharin, I. Batmanov, K. Lyubort, A. Okhotin, E. Sazhneva, "Formal languages over GF(2)", Language and Automata Theory and Applications (LATA 2018, Bar-Ilan near Tel Aviv, Israel, 9-11 April 2018), LNCS 10792, 68-79.

[4] Y. Bar-Hillel, M. Perles, E. Shamir, "On formal properties of simple phrasestructure grammars", Zeitschrift für Phonetik, Sprachwissenschaft und Kommunikationsforschung, 14 (1961), 143-177.

[5] N. Chomsky, M. P. Schützenberger, "Algebraic theory of context-free languages", Studies in Logic and the Foundations of Mathematics, 35 (1963), 118-161

[6] P. Flajolet, , "Analytic methods and ambiguity of context-free languages", Theoretical Computer Science, 49 (1987), 283-309 
[7] G. Christol, "Ensembles presque periodiques $k$-reconnaissables", Theoretical Computer Science, 9 (1979), 141-145.

[8] T. N. Hibbard, J. Ullian, "The independence of inherent ambiguity from complementedness among context-free languages", Journal of the ACM, 13 (1966), 588-593

[9] S. Ginsburg, H. G. Rice, "Two families of languages related to ALGOL", Journal of the ACM, 9 (1962), 350-371.

[10] S. Ginsburg, E. H. Spanier, "Bounded ALGOL-like languages", Transactions of the American Mathematical Society, Volume 113, number 2 (1964), 333-368

[11] S. Ginsburg, E. H. Spanier, "Semigroups, Presburger formulas, and languages", Pacific Journal of Mathematics, Volume 16, number 2 (1966), 285-296

[12] S. Ginsburg, J. Ullian, Ambiguity in context-free languages Journal of the ACM, 13 (1966), 62-89

[13] G. Jirásková, A. Okhotin, "Nondeterministic state complexity of positional addition", Journal of Automata, Languages and Combinatorics, 15:1-2 (2010), 121-133.

[14] I. Katsányi, "Sets of integers in different number systems and the Chomsky hierarchy", Acta Cybernetica, 15:2 (2001), 121-136.

[15] V. Makarov, A. Okhotin, "On the expressive power of GF(2)-grammars", SOFSEM 2019: Theory and Practice of Computer Science (Nový Smokovec, Slovakia, 27-30 January 2019), LNCS 11376, 310-323.

[16] A. Okhotin, E. Sazhneva, "State complexity of GF(2)-concatenation and GF(2)inverse on unary languages", Descriptional Complexity of Formal Systems (DCFS 2019, Kosice, Slovakia, 17-19 July 2019), to appear

[17] L. van Zijl, "On binary $\oplus$-NFAs and succinct descriptions of regular languages", Theoretical Computer Science, 328:1-2 (2004), 161-170. 


\section{Appendices}

\section{A What this part of the paper is about?}

In Sections A-C I present a longer, but a more elementary way to prove Theorems B.1 and C.1, through the use of algebraic expressions. In the end, it is no surprise that we will end up essentially reproving many of the algebraic statements I use in the main body of the paper, but in our particular special case.

Do manipulations with algebraic expressions provide any advantage at all over using Lemmas 3.2 and 4.1? Well, it $i s$ true that using algebraic expressions gives an effective way to transform a description of GF(2)-grammar that generates a subset of $a^{*} b^{*}$ or $a^{*} b^{*} c^{*}$ into a finite description of the corresponding power series. In a sense, many of the following results are can be seen as effective versions of qualitive Lemmas 3.2 and 4.1. However, I believe that modern algebraic geometry can prove much better quantitative versions of simple qualitive statements like those.

So, was it all in vain? I believe that the answer is "No". In a sense, this part of the paper "demystifies" its main body, because it more closely follows my original pattern of thinking. If you think that the new argument is too "magical", then, probably, this part of the paper is for you. What follows is, at least in my opinion, a very intuitive line of thinking. And in the end, we will be just one simple, but brilliant observation away from the much simpler final version of the argument.

The credit for this observation goes to an anonymous reviewer from MFCS 2020 conference. In short, and very paraphrased, they said "Aren't you essentially proving that $\frac{\sum \mathcal{A B}}{\operatorname{poly}(a, b)}$ describes a field? You can prove this in two sentences through standard results about field extensions". When pointed out, it sounds very simple, but, funnily enough, I never considered thinking about the equivalence between algebraic expressions $\frac{\sum \mathcal{A B}}{\sum \mathcal{A B}}$ and $\frac{\sum \mathcal{A B}}{\operatorname{poly}(a, b)}$ in such a way before!

So, in the end, you can see the following part of the paper as both an explanation for more "magical" part of the final argument, and as a story with a "plot twist" that almost everything I did was unnecessary. Personally, I think that knowing the twist only makes it more exciting.

\section{B Subsets of $a^{*} b^{*}$ through algebraic expressions}

\section{B.1 Algebraic expressions}

Let us define the meaning of words "Laurent series $f$ match algebraic expression $F$ ".

Informally, algebraic expressions are some formulas of symbols $\mathcal{A}, \mathcal{B}, \operatorname{poly}(a, b)$ and $\operatorname{rat}(a, b)$ that use additions, multiplications, divisions and "finite summation" operator, 
denoted by $\sum$.

Several examples of algebraic expressions: $\operatorname{rat}(a, b), \mathcal{B}, \sum \mathcal{A}, \frac{\sum \mathcal{A B}}{\sum \mathcal{A B}}, \sum \frac{\sum \mathcal{A B}}{\operatorname{rat}(a, b)}$, $\sum \mathcal{A B} \operatorname{rat}(a, b)$.

Definition. Laurent series $f$ match algebraic expression $F$ if and only if $f$ can be obtained from $F$ by substituting elements of $\operatorname{poly}(a, b), \operatorname{rat}(a, b), \mathcal{A}$ and $\mathcal{B}$ for coressponding symbols (not necessarily the same elements for the same symbols). The construct $\sum G$ corresponds to a finite, possibly empty, sum of Laurent series, with every summand matching $G$.

Example B.1. The set of Laurent series matching $\sum \mathcal{A B}$ is exactly the set of all power series representable as $A_{1} B_{1}+\ldots+A_{n} B_{n}$, where $n$ is any nonnegative integer, and $A_{i} \in \mathcal{A}$, $B_{i} \in \mathcal{B}$ for every $i$ from 1 to $n$ inclusive.

Example B.2. All rational functions in variables $a$ and $b$, and only them, match algebraic expressions $\operatorname{rat}(a, b)$ and $\frac{\operatorname{poly}(a, b)}{\operatorname{poly}(a, b)}$.

Example B.3. Laurent series match $\sum \frac{\sum \mathcal{A B}}{\operatorname{rat}(a, b)}$ if and only if it can be represented as a finite sum, where each summand can be represented as $\frac{A_{1} B_{1}+\ldots+A_{n} B_{n}}{p}$, for some nonnegative integer $n$ and some $A_{1}, \ldots, A_{n} \in \mathcal{A}, B_{1}, \ldots, B_{n} \in \mathcal{B}, p \in \operatorname{rat}(a, b)$, with an additional condition $p \neq 0$. The last condition is necessary because otherwise the denominator would be equal to zero and the fraction does not make sense.

Example B.4. Laurent series $f:=\sum_{n=0}^{+\infty} \sum_{m=0}^{+\infty}\left(a^{2^{n}} b^{2^{m}-10}+a^{2^{n}+10} b^{2^{m}+15}\right)$ match $\frac{\sum \mathcal{A B}}{\operatorname{poly}(a, b)}$, because $f=\frac{A_{1} B_{1}+A_{2} B_{2}}{b^{10}}$, zде $b^{10} \in \operatorname{poly}(a, b), A_{1}=\sum_{n=0}^{+\infty} a^{2^{n}} \in \mathcal{A}, B_{1}=\sum_{m=0}^{+\infty} b^{2^{m}} \in \mathcal{B}$, $A_{2}=\sum_{n=0}^{+\infty} a^{2^{n}+10} \in \mathcal{A}, B_{2}=\sum_{m=0}^{+\infty} b^{2^{m}+25} \in \mathcal{B}$.

Definition. Algebraic expressions $F$ and $G$ are equivalent (denoted by $F=G$ ), if subsets of Laurent series that match them coincide.

Some equivalencies follow directly from definitions and properties of classes poly $(a, b)$, $\operatorname{rat}(a, b), \mathcal{A}$ and $\mathcal{B}$. For example, $\sum \sum \mathcal{A B}=\sum \mathcal{A B}$, aforementioned $\operatorname{rat}(a, b)=\frac{\operatorname{poly}(a, b)}{\operatorname{poly}(a, b)}$, $\sum \mathcal{A} \mathcal{A}=\mathcal{A}$ and $\frac{\sum \mathcal{A B}}{\sum \mathcal{A B}}=\sum \frac{\mathcal{A B}}{\sum \mathcal{A B}}$.

Some equivalencies are not so trivial, like the equivalence $\frac{\sum \mathcal{A B}}{\sum \mathcal{A B}}=\frac{\sum \mathcal{A B}}{\operatorname{poly}(a, b)}$, which I shall establish later. 


\section{B.2 Switching to the algebraic track}

The purpose of this section is to prove the following intermediate result:

Lemma B.1. Assume that language $K \subset a^{*} b^{*}$ is described by a GF(2)-grammar. Then the corresponding power series asSeries $(K)$ match $\frac{\sum \mathcal{A B}}{\sum \mathcal{A B}}$.

Proof. Without loss of generality, the GF(2)-grammar that describes $K$ is in the Chomsky normal form [3, Theorem 5]. Moreover, let us assume that $K$ does not contain an empty string.

The language $a^{*} b^{*}$ is recognized by the following incomplete deterministic finite automaton $M$ : $M$ has two states $q_{a}$ and $q_{b}$, both accepting, and its transition function is $\delta\left(q_{a}, a\right)=q_{a}, \delta\left(q_{a}, b\right)=q_{b}, \delta\left(q_{b}, b\right)=q_{b}$.

Let us formally intersect the $\mathrm{GF}(2)$-grammar $G$ with regular language $a^{*} b^{*}$, recognized by the automaton $M$, using the construction of Bar-Hillel et al. [4] (it be used verbatim fo the GF(2) case [15, Section 6]). The language described by the GF(2)-grammar will not change, because it already was a subset of $a^{*} b^{*}$ before. The language described by the GF(2)-grammar will not change, because it is already a subset of $a^{*} b^{*}$.

The grammar will change considerably, however. Every nonterminal $C$ of the original grammar splits into three nonterminals: $C_{a \rightarrow a}, C_{a \rightarrow b}, C_{b \rightarrow b}$. Also a new starting nonterminal $S^{\prime}$ apears.

Every "normal" rule $C \rightarrow D E$ p splits into four rules: $C_{a \rightarrow a} \rightarrow D_{a \rightarrow a} E_{a \rightarrow a}, C_{a \rightarrow b} \rightarrow$ $D_{a \rightarrow a} E_{a \rightarrow b}, C_{a \rightarrow b} \rightarrow D_{a \rightarrow b} E_{b \rightarrow b}$ and $C_{b \rightarrow b} \rightarrow D_{b \rightarrow b} E_{b \rightarrow b}$.

The following happens with "final" rules: $C \rightarrow b$ turns into two rules $C_{a \rightarrow b} \rightarrow b$ and $C_{b \rightarrow b} \rightarrow b$, and $C \rightarrow a$ turns into one rule $C_{a \rightarrow a} \rightarrow a$. Finally, two more rules appear: $S^{\prime} \rightarrow S_{a \rightarrow a}$ and $S^{\prime} \rightarrow S_{a \rightarrow b}$.

What do nonterminals of the new GF(2)-grammar correspond to? The state $C_{a \rightarrow a}$ corresponds to the strings $w \in\{a, b\}^{*}$ that are derived from the nonterminal $C$ of the original $\mathrm{GF}(2)$-grammar and make $M$ go from the state $q_{a}$ to itself. Formally speaking, $w \in L\left(C_{a \rightarrow a}\right)$ if and only if $w \in L(C)$ and $\delta\left(q_{a}, w\right)=q_{a}$. Similarly, $w \in L\left(C_{a \rightarrow b}\right)$ if and only if $w \in L(C)$ and $\delta\left(q_{a}, w\right)=q_{b}$. Finally, $w \in L\left(C_{b \rightarrow b}\right)$ if and only if $w \in L(C)$ and $\delta\left(q_{b}, w\right)=q_{b}$.

By looking more closely on the transitions of $M$, we can see that $\delta\left(q_{a}, w\right)=q_{a}$ if and only if $w$ consists only of letters $a$, in other words, if and only if $w \in a^{*}$. Similarly, $\delta\left(q_{b}, w\right)=q_{b}$ if and only if $w \in b^{*}$, and $\delta\left(q_{a}, w\right)=q_{b}$ if and only if $w \in a^{*} b^{+}$.

Every language $L\left(C_{a \rightarrow a}\right)$ is a 2-automatic language over a unary alphabet $\{a\}$. Indeed, every parse tree of $C_{a \rightarrow a}$ contains only nonterminals of type $a \rightarrow a$. Therefore only symbol $a$ can occur as a terminal in the parse tree. So, $L\left(C_{a \rightarrow a}\right)$ is described by some $\mathrm{GF}(2)$-grammar over an alphabet $\{a\}$, and is therefore 2-automatic. Similarly, all languages $L\left(C_{b \rightarrow b}\right)$ are 2-automatic over the alphabet $\{b\}$. Then, by Christol's theorem, asSeries $\left(L\left(C_{a \rightarrow a}\right)\right) \in \mathcal{A}$ and $\operatorname{asSeries}\left(L\left(C_{b \rightarrow b}\right)\right) \in \mathcal{B}$.

How do the languages $L\left(C_{a \rightarrow b}\right)$ look like? Let us look at the rules $C_{a \rightarrow b} \rightarrow D_{a \rightarrow a} E_{a \rightarrow b}$ and $C_{a \rightarrow b} \rightarrow D_{a \rightarrow b} E_{b \rightarrow b}$. These rules can be interpreted in the following way: when starting 
a parse from nonterminal $C_{a \rightarrow b}$, we can append a language from $\mathcal{A}$ from the left and go to $E_{a \rightarrow b}$ or append a language from $\mathcal{B}$ from the right and go to $D_{a \rightarrow b}$.

What can we say about $K$ ? By definition, $K=L(S)=L\left(S^{\prime}\right)=L\left(S_{a \rightarrow a}\right) \triangle L\left(S_{a \rightarrow b}\right)$. We can forget about the language $L\left(S_{a \rightarrow a}\right)$ : it is from the class $\mathcal{A}$, and $L\left(S_{a \rightarrow b}\right)$ is from much more complicated class, that will "absorb" $\mathcal{A}$ in the end.

The languages $L\left(C_{a \rightarrow b}\right)$ for each nonterminal $C_{a \rightarrow b}$ of the new grammar satisfy the following system of language equations:

$$
L\left(C_{a \rightarrow b}\right)=\operatorname{end}\left(C_{a \rightarrow b}\right) \triangle \bigwedge_{C \rightarrow D E}\left(L\left(D_{a \rightarrow a}\right) \odot L\left(E_{a \rightarrow b}\right)\right) \triangle\left(L\left(D_{a \rightarrow b}\right) \odot L\left(E_{b \rightarrow b}\right)\right)
$$

Here, the summation happens over all rules $C \rightarrow D E$ for each nonterminal $C$ of the original grammar, and end $\left(C_{a \rightarrow b}\right)$ is either $\{b\}$ or $\varnothing$, depending on whether or not there is a rule $C_{a \rightarrow b} \rightarrow b$ in the new grammar.

Look more closely at the system (7). In all GF(2)-concatenations that appear in its right-hand side either the first language is a subset of $a^{*}$, or the second language is a subset of $b^{*}$. Hence, we can apply the Lemma 3.1.

Denote $\operatorname{asSeries}\left(L\left(C_{a \rightarrow b}\right)\right)$ by $\operatorname{Center}(C), \quad \operatorname{asSeries}\left(L\left(C_{a \rightarrow a}\right)\right)$ by $\operatorname{Left}(C)$, $\operatorname{asSeries}\left(L\left(C_{b \rightarrow b}\right)\right)$ by $\operatorname{Right}(C)$ and asSeries $\left(\operatorname{end}\left(C_{a \rightarrow b}\right)\right)$ by final $(C)$ for brevity.

Applying asSeries to the both sides of (7) gives us the following system of equations over formal power series:

$$
\operatorname{Center}(C)=\operatorname{final}(C)+\sum_{C \rightarrow D E} \operatorname{Left}(D) \operatorname{Center}(E)+\operatorname{Center}(D) \operatorname{Right}(E)
$$

Let us look at this system as a system of $\mathbb{F}_{2}[[a, b]]$-linear equations over variables Center $(C)=\operatorname{asSeries}\left(L\left(C_{a \rightarrow b}\right)\right)$ for every nonterminal $C$ of the original $\mathrm{GF}(2)$-grammar.

We will consider final $(C), \operatorname{Left}(C)$ and $\operatorname{Right}(C)$ to be the coefficients of the system. While we do not know their exact values, the following is $\operatorname{known}: \operatorname{final}(C)$ is 0 or $b$, $\operatorname{Left}(C) \in \mathcal{A}$ as a formal power series that corresponds to a 2-automatic language over an alphabet $\{a\}$ and, similarly, $\operatorname{Right}(C) \in \mathcal{B}$.

Denote is the number of nonterminals in the original GF(2)-grammar by $n$, (so there are $n$ nonterminals of type $a \rightarrow b$ in the new GF(2)-grammar), a column vector of values Center $(C)$ by $x$ and a column vector of values final $(C)$ in the same order by $f$. Let us fix the numeration of nonterminals $C$ of the old GF(2)-grammar. After that, we can use them as "numbers" of rows and columns of matrices.

Let $I$ be an identity matrix of dimension $n \times n, A$ be a $n \times n$ matrix with the sum of Left $(D)$ over all rules $C \rightarrow D E$ of the original grammar standing on the intersection of $C$-th row and $E$-th column:

$$
A_{C, E}:=\sum_{C \rightarrow D E} \operatorname{Left}(D)
$$

Similarly, let $B$ be a $n \times n$ matrix with

$$
B_{C, D}:=\sum_{C \rightarrow D E} \operatorname{Right}(E)
$$


Then the equation system (8) can be rewritten as $x=f+(A+B) x$ in the matrix form. In other words, $(A+B+I) x=f$.

We have already proven earlier that $\operatorname{Center}(C)$ is a solution of this system. Our plan is to prove that there is exactly one solution to this system and express it in some form. Then, in particular, we will find some expression for asSeries $\left(L\left(S_{a \rightarrow b}\right)\right)=\operatorname{Center}(S)$.

This system has exactly one solution if and only if $\operatorname{det}(A+B+I) \neq 0$. If $\operatorname{det}(A+B+I) \neq$ 0 , then, by Cramer's formula, every entry of the solution, including $\operatorname{Center}(S)$ can be written as

$$
\frac{\operatorname{det}(A+B+I, \text { but with one of the columns replaced by } f)}{\operatorname{det}(A+B+I)}
$$

It remains to establish three things: that $\operatorname{det}(A+B+I)$ matches $\sum \mathcal{A B}$, that $\operatorname{det}(A+B+I$, but with one of the columns replaced by $f)$ matches $\sum \mathcal{A B}$, independently of the replaced column and that $\operatorname{det}(A+B+I) \neq 0$.

Let us prove the first two statements at the same time. Every entry of $A+B+I$ matches $\mathcal{A}+\mathcal{B}$ because of the equations (9)-(10). Indeed, every entry of $A$ matches $\sum \mathcal{A}=\mathcal{A}$, every entry of $B$ match $\mathcal{B}$, and entries of $I$ are ones and zeroes that lie in both $\mathcal{A}$ and $\mathcal{B}$. This property will not disappear, if you replace every column of the matrix by $f$ : all entries of $f$ are equal to 0 or $b$, so they match $\mathcal{B}$, let alone $\mathcal{A}+\mathcal{B}$.

Now, let us prove that the determinant of the matrix with every entry matching $\mathcal{A}+\mathcal{B}$ match $\sum \mathcal{A B}$. Indeed, if we use the exact formula for determinant with $n$ ! summands, we get that match $\sum \underbrace{(\mathcal{A}+\mathcal{B}) \cdot \ldots \cdot(\mathcal{A}+\mathcal{B})}_{n \text { times }}$. By expanding the brackets and using the fact that $\mathcal{A} \mathcal{A}=\mathcal{A}$ and $\mathcal{B B}=\mathcal{B}$, we see that the determinant match $\sum \mathcal{A B}$.

It remains to prove that $\operatorname{det}(A+B+I) \neq 0$. Let us prove a stronger statement: that the power series $\operatorname{det}(A+B+I) \in \mathbb{F}_{2}[[a, b]]$ is invertible, that is, its coefficient при $a^{0} b^{0}$ is equal to 1 (for purposes of this proof, you may think that it is the definition of invertibility).

Notice that finite product of power series is invertible if and only if each multiplier is invertible. Also a finite sum of power series with exactly one invertible summand is invertible.

Because the new GF(2)-grammar is also in Chomsky's normal form, all languages $L\left(C_{a \rightarrow a}\right)$ and $L\left(C_{b \rightarrow b}\right)$ do not contain an empty word. Therefore, all series $\operatorname{Left}(C)$ and Right $(C)$ are invertibe. Therefore, by equations (9)-(10), all entries of $A+B$ are invertible. It follows that exactly the diagonal entries of $A+B+I$ are invertible: they are obtained by adding one to invertible series, and other entries of $A+B+I$ coincide with the same entries of $A+B$.

Let us use the formula for $\operatorname{det}(A+B+I)$ with $n$ ! summands again. Exactly one summand is invertible: the one that corresponds to the identity permutation. Indeed, all other summands have at least one nondiagonal, therefore, non-invertible, element. In the summand that corresponds to identity permutation, all multiplies are diagonal entries of $A+B+I$, hence invertible power series.

We just proved that $\operatorname{det}(A+B+I)$ is invertible. In particular, $\operatorname{det}(A+B+I) \neq 0$.

We have proved that asSeries $\left(L\left(S_{a \rightarrow b}\right)\right)$ match $\frac{\sum \mathcal{A B}}{\sum \mathcal{A B}}$. Then asSeries $(K)=$ 
$\operatorname{asSeries}\left(L\left(S^{\prime}\right)\right)=\operatorname{asSeries}\left(L\left(S_{a \rightarrow a}\right)+\operatorname{asSeries}\left(L\left(S_{a \rightarrow b}\right)\right) \operatorname{match} \mathcal{A}+\frac{\sum \mathcal{A B}}{\sum \mathcal{A B}}=\frac{\sum \mathcal{A B}}{\sum \mathcal{A B}}\right.$. The last equivalence holds, because wе can привести сумму к общему знаменателю and obtain $\mathcal{A} \sum \mathcal{A B}+\sum \mathcal{A B}=\sum \mathcal{A B}$ in the numerator.

\section{B.3 Algebraic manipulations}

The purpose of this section is to prove the following theorem:

Theorem B.1. If $L \subset a^{*} b^{*}$ is described by a GF(2)-grammar. Then the corresponding power series asSeries $(L)$ match $\frac{\sum \mathcal{A B}}{\operatorname{poly}(a, b)}$.

In the previous section, we have already moved to this goal, by dealing with languagetheoretic details. Now, we want to use some algebraic manipulations. Theorem B.1 would follow from the Lemma B.1 and the following lemma:

Lemma B.2. Algebraic expressions $\frac{\sum \mathcal{A B}}{\sum \mathcal{A B}}$ and $\frac{\sum \mathcal{A B}}{\operatorname{poly}(a, b)}$ are equivalent.

Proof. It is evident that the algebraic expression $\frac{\sum \mathcal{A B}}{\sum \mathcal{A B}}$ is not weaker than $\frac{\sum \mathcal{A B}}{\operatorname{poly}(a, b)}$, because $\sum \mathcal{A B}$ is not weaker than poly $(a, b)$. Indeed, every polynomial is a finite sum of monomials of type $a^{n} b^{m}$, and each such monomial match $\mathcal{A B}$, because $a^{n} \in \mathcal{A}$ and $b^{m} \in \mathcal{B}$.

The converse implication, namely that $\frac{\sum \mathcal{A B}}{\sum \mathcal{A B}}$ is not stronger than $\frac{\sum \mathcal{A B}}{\text { poly }(a, b)}$, is more interesting. Suppose that some formal power series $f$ match $\frac{\sum \mathcal{A B}}{\sum \mathcal{A B}}$. Then it also match $\sum \frac{\mathcal{A B}}{\sum \mathcal{A B}}$. Let us show that each of the summands match $\frac{\sum \mathcal{A B}}{\operatorname{poly}(a, b)}$, then the whole sum match $\sum \frac{\sum \mathcal{A B}}{\operatorname{poly}(a, b)}=\frac{\sum \mathcal{A B}}{\operatorname{poly}(a, b)}$, as intended.

Indeed, suppose that we have some Laurent series matching the expression $\frac{\mathcal{A B}}{\sum \mathcal{A B}}$. Then, by definition of "matching algebraic expression", these series are of the type $\frac{A_{0} B_{0}}{A_{1} B_{1}+A_{2} B_{2}+\ldots+A_{n} B_{n}}$, where $n$ is a positive integer, and $A_{i} \in \mathcal{A}, B_{i} \in \mathcal{B}$ for every $i$ from 0 to $n$ inclusive. Moreover, this expression makes sense, meaning that $A_{1} B_{1}+\ldots+A_{n} B_{n} \neq 0$.

We still have not used that $\mathcal{A}$ and $\mathcal{B}$ are exactly the sets of algebraic power series, and not just some subsets of $\mathbb{F}_{2}[[a]]$ and $\mathbb{F}_{2}[[b]]$ that are closed under addition. Let us use that.

More exactly, we want to get rid of difficult expression in the numerator by rewritting $\frac{1}{A_{1} B_{1}+\ldots+A_{n} B_{n}}$, that is, $\left(A_{1} B_{1}+\ldots+A_{n} B_{n}\right)^{-1}$, as a finite rat $(a, b)$-linear combination of nonnegative powers of $A_{1} B_{1}+\ldots+A_{n} B_{n}$. 
The least painful way to do so is to find a nontrivial rat $(a, b)$-linear dependence between nonnegative powers of $A_{1} B_{1}+\ldots+A_{n} B_{n}$ and then get the required expression from it. It still is not very easy, see below for details.

Because every $A_{i}$ is an algebraic power series of variable $a$ over the ring $\mathbb{F}_{2}[a]$, it also is an algebraic power series in variables $a$ and $b$ over the field $\operatorname{rat}(a, b)$ : the same polynomial equation will suffice to show that.

We will need a few technical lemmas:

Lemma B.3. Suppose that $f \in \mathbb{F}_{2}[[a, b]]$ is a solution to a polynomial equation of degree $d$ with coefficients from $\operatorname{rat}(a, b)$. Then, for every $m \geqslant d, f^{m}$ can be represented as a $\operatorname{rat}(a, b)$-linear combination of $f^{m-1}, f^{m-2}, \ldots, f^{m-d}$.

Proof. Indeed, by conditions of the lemma, $\sum_{i=0}^{d} p_{i} f^{i}=0$ for some $p_{i} \in \operatorname{rat}(a, b)$. Moreover, $p_{d} \neq 0$, because the degree of the equation is exactly $d$. Divide both sides by $p_{d}$ and move $f^{d}$ to the right-hand side: $\sum_{i=0}^{d-1} \frac{p_{i}}{p_{d}} f^{i}=f^{d}$. Multiply both sides by $f^{m-d}$ : $\sum_{j=m-d}^{m-1} \frac{p_{j-(m-d)}}{p_{d}} f^{j}=f^{m}$, exactly a representation of $f^{m}$ as a rat $(a, b)$-linear combination of $f^{m-1}, f^{m-2}, \ldots, f^{m-d}$.

Lemma B.4. Suppose that $f \in \mathbb{F}_{2}[[a, b]]$ is a root of polynomial equation of degree $d$ with coefficients from $\operatorname{rat}(a, b)$. Then, for every $m \geqslant 0, f^{m}$ can be represented as $\operatorname{rat}(a, b)$ linear combination of $f^{d-1}, f^{d-2}, \ldots, f^{0}$. In other words, all nonnegative powers of $f$ are in $\operatorname{rat}(a, b)$-linear space generated by $f^{0}, f^{1}, \ldots, f^{d-1}$.

Proof. Induction over $m$. Denote the $\operatorname{rat}(a, b)$-linear space, generated by $f^{0}, f^{1}, \ldots, f^{d-1}$ by $L$. The statement is trivially true for $m<d$, because $f^{m}$ is one of generators of $L$.

Now, suppose that we want to prove the statement of the lemma for some $m \geqslant d$. By induction hypothesis, $f^{m-1}, f^{m-2}, \ldots, f^{m-d}$ all lie in $L$. By the previous lemma, $f^{m}$ can be represented as a rat $(a, b)$-linear combination of $f^{m-1}, f^{m-2}, \ldots, f^{m-d}$. Therefore, $f^{m}$ lies in $L$ as a finite $\operatorname{rat}(a, b)$-linear combination of elements of $L$.

Lemma B.5. There is no infinite subset of monomials in variables $A_{i}$ and $B_{i}$, that is linearly independent over $\operatorname{rat}(a, b)$. In other words, $\operatorname{rat}(a, b)$-linear space generated by all values of polynomials in variables $A_{1}, A_{2}, \ldots, A_{n}$ and $B_{1}, B_{2}, \ldots, B_{n}$ with coefficients from $\operatorname{rat}(a, b)$ is finite-dimensional.

Proof. Because $A_{i} \in \mathcal{A}$ for every $i$ from 1 to $n$ inclusive, there are some $\ell_{i}$ such that $A_{i}$ is a root of degree- $d$ polynomial equation with coefficients from $\operatorname{rat}(a, b)$. Similarly, denote by $r_{i}$ the degrees of polynomial equations for $B_{i}$.

Let us try to represent expression $A_{1}^{j_{1}} \ldots A_{n}^{j_{n}} \cdot B_{1}^{k_{1}} \ldots B_{n}^{k_{n}}$ for some nonnegative $j_{s}$ and $k_{s}$ as a $\operatorname{rat}(a, b)$-linear combination of similar expressions with small degrees.

Indeed, by previous lemma, every $A_{s}^{j_{s}}$ is a rat $(a, b)$-linear combination of $A_{s}^{0}, A_{s}^{1}, \ldots, A_{s}^{\ell_{s}-1}$. Similarly, every $B_{s}^{k_{s}}$ is a rat $(a, b)$-linear combination of 
$B_{s}^{0}, B_{s}^{1}, \ldots, B_{s}^{r_{s}-1}$. Represent $A_{1}^{j_{1}} \ldots A_{n}^{j_{n}} \cdot B_{1}^{k_{1}} \ldots B_{n}^{k_{n}}$ as a product of such linear combination and expand all brackets. The result is some rat $(a, b)$-linear combination of expressions $A_{1}^{x_{1}} \ldots A_{n}^{x_{n}} \cdot B_{1}^{y_{1}} \ldots B_{n}^{y_{n}}$, but with $0 \leqslant x_{s}<\ell_{s}$ and $0 \leqslant y_{s}<r_{s}$.

Let $L$ be a $\operatorname{rat}(a, b)$-linear space, generated by all products of type $A_{1}^{x_{1}} A_{2}^{x_{2}} \ldots A_{n}^{x_{n}}$. $B_{1}^{y_{1}} \ldots B_{n}^{y_{n}}$, where $0 \leqslant x_{s}<\ell_{s}$ and $0 \leqslant y_{s}<r_{s}$ for all $s$ from 1 to $n$ inclusive. This space is generated by $\ell_{1} \ell_{2} \ldots \ell_{n} \cdot r_{1} r_{2} \ldots r_{n}$ elements and therefore is finite-dimensional.

We already established that every monomial $A_{1}^{j_{1}} \ldots A_{n}^{j_{n}} \cdot B_{1}^{k_{1}} \ldots B_{n}^{k_{n}}$ is a $\operatorname{rat}(a, b)$-linear combination of elements of $L$ (moreover, exactly the elements that were $L$ 's generators), therefore it lies in $L$. Then, every polynomial expression in variables $A_{s}$ and $B_{s}$ lies in $L$, as a linear combination of monomials that lie in $L$.

Because the space of all polynomial expression of $A_{i}$ and $B_{i}$ is finite-dimensional, the space generated by nonnegative powers of $A_{1} B_{1}+\ldots+A_{n} B_{n}$ also is. Therefore, there exists a nontrivial $\operatorname{rat}(a, b)$-linear dependence between nonnegative powers of $A_{1} B_{1}+\ldots+A_{n} B_{n}$. In other words, there is some nonnegative integer $d$ and rational functions $p_{0}, p_{1}, \ldots, p_{d} \in$ $\operatorname{rat}(a, b)$, not all equal to zero, such that $\sum_{i=0}^{d} p_{i}\left(A_{1} B_{1}+\ldots+A_{n} B_{n}\right)^{i}=0$. Let us find an expression of $\left(A_{1} B_{1}+\ldots+A_{n} B_{n}\right)^{-1}$ through nonnegative powers of $A_{1} B_{1}+\ldots+A_{n} B_{n}$ with that knowledge.

Indeed, let us take the smallest such $j$ that $p_{j} \neq 0$. It exists, because not all $p_{i}$ are equal to zero. Then our equation can be rewritten as $\sum_{i=j}^{d} p_{i}\left(A_{1} B_{1}+\ldots+A_{n} B_{n}\right)^{i}=0$, because $p_{0}=p_{1}=\ldots=p_{j-1}=0$ anyways. By dividing both sides by $p_{j}\left(A_{1} B_{1}+\ldots+A_{n} B_{n}\right)^{j+1} \neq 0$, we obtain $\sum_{i=j}^{d} \frac{p_{i}}{p_{j}}\left(A_{1} B_{1}+\ldots+A_{n} B_{n}\right)^{i-j-1}=0$. All powers of $A_{1} B_{1}+\ldots+A_{n} B_{n}$ from $(-1)$ st to $(d-j-1)$-st are here with some coefficients, the coefficient before $(-1)$-st power is $p_{j} / p_{j}=1$. By moving all powers, except $(-1)$-st to the right-hand side, we obtain $\left(A_{1} B_{1}+\right.$ $\left.\ldots+A_{n} B_{n}\right)^{-1}=\sum_{i=j+1}^{d} \frac{p_{i}}{p_{j}}\left(A_{1} B_{1}+\ldots+A_{n} B_{n}\right)^{i-j-1}=\sum_{i=0}^{d-j-1} \frac{p_{i+j+1}}{p_{j}}\left(A_{1} B_{1}+\ldots+A_{n} B_{n}\right)^{i}$.

Therefore, $\left(A_{1} B_{1}+\ldots+A_{n} B_{n}\right)^{-1}$ match $\sum \operatorname{rat}(a, b) \mathcal{A B}$ (to understand that, expand all brackets in the right-hand side). Therefore, $\frac{A_{0} B_{0}}{A_{1} B_{1}+\ldots+A_{n} B_{n}}$ match $\mathcal{A B}$. $\sum \operatorname{rat}(a, b) \mathcal{A B}=\sum \operatorname{rat}(a, b) \mathcal{A B}$. We are almost done!

Remark. Generally speaking, $\operatorname{rat}(a, b)$ cannot be split into two parts with first being "absorbed" by $\mathcal{A}$ and the second being "absorbed" by $\mathcal{B}$. Keep the following example in the head: $1+a b$. It is not hard to prove that $1+a b$ is not a product of a multiplier depending only on $a$ and a multiplier depending only on $b$.

As we understood earlier, every Laurent series matching $\frac{\mathcal{A B}}{\sum \mathcal{A B}}$ also match $\sum \operatorname{rat}(a, b) \mathcal{A B}$. Then all Laurent series matching $\frac{\sum \mathcal{A B}}{\sum \mathcal{A B}}$ also match $\sum \sum \operatorname{rat}(a, b) \mathcal{A B}=$ 
$\sum \operatorname{rat}(a, b) \mathcal{A B}$. Finally, by adding the fractions up, every Laurent series matching $\sum \operatorname{rat}(a, b) \mathcal{A B}$ match $\frac{\sum \operatorname{poly}(a, b) \mathcal{A B}}{\operatorname{poly}(a, b)}=\frac{\sum \mathcal{A B}}{\operatorname{poly}(a, b)}$.

\section{Subsets of $a^{*} b^{*} c^{*}$ through algebraic expressions}

The language $\left\{a^{n} b^{n} c^{n} \mid n \geqslant 0\right\}$ is, probably, the most famous example of a simple language that is not described by any ordinary grammar. It is reasonable to assume that it is not described by a GF(2)-grammar as well. Let us prove that.

We will do more than that and will actually establish some property that all GF(2)grammatical subsets of $a^{*} b^{*} c^{*}$ have, but $\left\{a^{n} b^{n} c^{n} \mid n \geqslant 0\right\}$ does not. Most steps of the proof will be analogous to the two-letter case.

There is a natural one-to-one correspondence between subsets of $a^{*} b^{*} c^{*}$ and formal power series in variables $a, b$ and $c$ over field $\mathbb{F}_{2}$. Indeed, for every set $S \subset \mathbb{N}_{0}^{3}$, we can identify the language $\left\{a^{n} b^{m} c^{k} \mid(n, m, k) \in S\right\} \subset a^{*} b^{*} c^{*}$ with the formal power series $\sum_{(n, m, k) \in S} a^{n} b^{m} c^{k}$. Denote this corres'pondence by asSeries: $2^{a^{*} b^{*} c^{*}} \rightarrow \mathbb{F}_{2}[[a, b, c]]$. Then, $\operatorname{asSeries}(L \triangle K)=\operatorname{asSeries}(L)+\operatorname{asSeries}(K)$. In other words, the symmetric difference of languages corresponds to the sum of formal power series.

The product of formal power series corresponds to commutative GF(2)-concatenation of languages:

$$
\begin{aligned}
K \odot_{\text {comm }} L=\left\{a^{n} b^{m} c^{k} \mid \text { number of representations } n\right. & =n_{1}+n_{2}, m=m_{1}+m_{2} \text { and } \\
k=k_{1}+k_{2}, \text { where } a^{n_{1}} b^{m_{1}} c^{k_{1}} & \left.\in K \text { and } a^{n_{2}} b^{m_{2}} c^{k_{2}} \in L, \text { is odd }\right\}
\end{aligned}
$$

Commutative $\mathrm{GF}(2)$-concatenation is commutative and corresponds to the product of formal power series: asSeries $\left(K \odot_{\text {comm }} L\right)=\operatorname{asSeries}(K) \cdot \operatorname{asSeries}(L)$.

Commutative GF(2)-concatenation of languages $K$ and $L$ coincides with normal GF(2)concatenation in the three following important cases: when $K$ is a subset of $a^{*}$, when $K$ is a subset of $a^{*} b^{*}$ and $L$ is a subset of $b^{*} c^{*}$, and, finally, when $L$ is a subset of $c^{*}$. Indeed, in every of these three cases, symbols "are in the correct order": if $u \in K$ and $v \in L$, then $u v \in a^{*} b^{*} c^{*}$.

However, we cannot insert character $b$ in the middle of the string: if $K$ is a subset of $b^{*}$ and $L$ is a subset of $a^{*} b^{*} c^{*}$, then $K \odot L$ and $K \odot$ comm $L$ do not have to coincide, because $K \odot L$ does not even have to be a subset of $a^{*} b^{*} c^{*}$.

The "work plan" will remain the same as in the previous section: we will switch to algebraic track first and then we simplify the obtained expression.

An attentive reader may ask two questions:

1. Why it is logical to expect that the language $\left\{a^{n} b^{n} c^{n} \mid n \geqslant 0\right\}$ is not described by a $\mathrm{GF}(2)$-grammar, but a similar language $\left\{a^{n} b^{n} \mid n \geqslant 0\right\}$ is?

2. Why the proof will work out for $\left\{a^{n} b^{n} c^{n} \mid n \geqslant 0\right\}$, but not for a regular language $\left\{(a b c)^{n} \mid n \geqslant 0\right\}$, despite these languages having the same "commutative images"? 
I will try to give an answer.

1. Simply speaking, the reason is the same as for the ordinary grammars. On intuitive levels, both ordinary grammars and GF(2)-grammars permit a natural way to "capture" the events that happen with any two letters in subsets of $a^{*} b^{*} c^{*}$, but not all three letters at the same time. A rigourous result that corresponds to this intuitive limitation of ordinary grammars was proven by Ginsburg and Spanier [10, Theorem 2.1]. Theorem C.1 is an analogue for GF(2)-grammars.

2. This argument only implies that any proof that relies solely on commutative images is going to fail. The real proof is more subtle. For example, it will also use the fact that $\left\{a^{n} b^{n} c^{n} \mid n \geqslant 0\right\}$ is a subset of $a^{*} b^{*} c^{*}$.

While the proof uses commutative images, it uses them very accurately, always making sure to satisfy the conditions of Lemma 3.1. In particular, I never consider GF(2)-concatenations $K \odot L$, where $K$ is a subset of $b^{*}$ and $L$ is an arbitrary subset of $a^{*} b^{*} c^{*}$.

Avoiding this situation is impossible for language $\left\{(a b c)^{n} \mid n \geqslant 0\right\}$, because in the word $a b c a b c$ from this language the letters "appear in the wrong order".

Denote the set of algebraic power series of variable $c$ by $\mathcal{C}$, the set of polynomials in variables $a$ and $c$ by $\operatorname{poly}(a, c)$, et cetera.

The definitions of algebraic expression stays mostyly the same, but now "characters" $\mathcal{C}$, $\operatorname{poly}(a, c), \operatorname{poly}(b, c), \operatorname{poly}(a, b, c), \operatorname{rat}(a, c), \operatorname{rat}(b, c)$ and $\operatorname{rat}(a, b, c)$ may appear alonhside the old $\mathcal{A}, \mathcal{B}$ and $\operatorname{poly}(a, b)$.

\section{C.1 Switching to the algebraic track}

Our goal for this subsection is to establish the following lemma:

Lemma C.1. Suppose that $K \subset a^{*} b^{*} c^{*}$ is described by a GF(2)-grammar. Then the corresponding formal power series asSeries $(K)$ match algebraic expression $\frac{\sum \mathcal{A B C}}{\operatorname{poly}(a, b) \operatorname{poly}(b, c) \cdot \sum \mathcal{A C}}$.

Proof. The proof is mostly the same as the proof of Lemma B.1.

Without loss of generality, GF(2)-grammar $G$ that describes $K$ is in Chomsky's normal form. Also we can assume that $K$ does not contain the empty string.

The language $a^{*} b^{*} c^{*}$ is accepted by the following incomplete deterministic finite automaton $M$. Firstly, $M$ has three states $q_{a}, q_{b}$ and $q_{c}$, all accepting. Secondly, its transition function $\delta$ is defined as $\delta\left(q_{a}, a\right)=q_{a}, \delta\left(q_{a}, b\right)=q_{b}, \delta\left(q_{a}, c\right)=q_{c}, \delta\left(q_{b}, b\right)=q_{b}, \delta\left(q_{b}, c\right)=$ $q_{c}, \delta\left(q_{c}, c\right)=q_{c}$.

Intersect the $\mathrm{GF}(2)$-grammar $G$ formally with regular language $a^{*} b^{*} c^{*}$, recognized by $M$. Because $L(G)=K$ was a subset of $a^{*} b^{*} c^{*}$ anyway, the described language will not 
change. Each nonterminal $C$ of the original grammar will split into six nonterminals $C_{a \rightarrow a}$, $C_{a \rightarrow b}, C_{a \rightarrow c}, C_{b \rightarrow b}, C_{b \rightarrow c}, C_{c \rightarrow c}$. Also, a new starting nonterminal $S^{\prime}$ will appear.

Every "normal" rule $C \rightarrow D E$ will split into rules $C_{a \rightarrow a} \rightarrow D_{a \rightarrow a} E_{a \rightarrow a}, C_{a \rightarrow b} \rightarrow$ $D_{a \rightarrow a} E_{a \rightarrow b}, C_{a \rightarrow b} \rightarrow D_{a \rightarrow b} E_{b \rightarrow b}, C_{a \rightarrow c} \rightarrow D_{a \rightarrow a} E_{a \rightarrow c}, C_{a \rightarrow c} \rightarrow D_{a \rightarrow b} E_{b \rightarrow c}, C_{a \rightarrow c} \rightarrow$ $D_{a \rightarrow c} E_{c \rightarrow c}, C_{b \rightarrow c} \rightarrow D_{b \rightarrow b} E_{b \rightarrow c}, C_{b \rightarrow c} \rightarrow D_{b \rightarrow c} E_{c \rightarrow c}$, and $C_{c, c} \rightarrow D_{c \rightarrow c} E_{c \rightarrow c}$. Less horrifying than it looks, because most of these rules will not be interesting to us in the slightest.

A "final" rule $C \rightarrow a$ will turn into a rule $C_{a \rightarrow a} \rightarrow a$. Similarly, rule $C \rightarrow b$ will split into two rules $C_{a \rightarrow b} \rightarrow b$ and $C_{b \rightarrow b} \rightarrow b$, and rule $C \rightarrow c$ will split into three rules $C_{a \rightarrow c} \rightarrow c$, $C_{b \rightarrow c} \rightarrow c$ and $C_{c \rightarrow c} \rightarrow c$.

Finally, three new rules will appear: $S^{\prime} \rightarrow S_{a \rightarrow a}, S^{\prime} \rightarrow S_{b \rightarrow b}, S^{\prime} \rightarrow S_{c \rightarrow c}$.

Nonterminal $C_{x \rightarrow y}$ of the new $\mathrm{GF}(2)$-grammar, where $x, y \in\{a, b, c\}$, corresponds to exactly such strings from $L(C)$ that move the automaton $M$ from the state $q_{x}$ to the state $q_{y}$.

By looking more closely at the transitions of automaton $M$, we can see that any string that makes $M$ go from $q_{a}$ to $q_{c}$ is from $a^{*} b^{*} c^{*}$, any string that makes $M$ go from $q_{b}$ to $q_{c}$ is from $b^{*} c^{*}$, et cetera. In particular, in all new "normal" rules $\mathrm{GF}(2)$-concatenations coincide with corresponding commutative $\mathrm{GF}(2)$-concatenations, because the letters $a, b$ and $c$ appear in that exact order in the words.

As already mentioned, most of the new rules are not interesting, because we already know, how the languages $L\left(C_{a \rightarrow a}\right), L\left(C_{a \rightarrow b}\right), L\left(C_{b \rightarrow b}\right), L\left(C_{b \rightarrow c}\right)$ and $L\left(C_{c \rightarrow c}\right)$ look like. More specifically, the corresponding formal power series match algeebraic expressions $\mathcal{A}$, $\frac{\sum \mathcal{A B}}{\operatorname{poly}(a, b)}, \mathcal{B}, \frac{\sum \mathcal{B C}}{\text { poly }(b, c)}$ and $\mathcal{C}$ respectively.

Therefore, we are only interested in nonterminals of the type $a \rightarrow c$. The rule $X \rightarrow Y Z$ of the original GF(2)-grammar $G$ produces three rules for $X_{a \rightarrow c}: X_{a \rightarrow c} \rightarrow Y_{a \rightarrow c} Z_{c \rightarrow c}$, $X_{a \rightarrow c} \rightarrow Y_{a \rightarrow b} Z_{b \rightarrow c}$ and $X_{a \rightarrow c} \rightarrow Y_{a \rightarrow a} Z_{a \rightarrow c}$. The first and last rule relate $L\left(X_{a \rightarrow c}\right)$ to other nonterminals of type $a \rightarrow c$, and the second rule just outright tells us that we can replace $X_{a \rightarrow c}$ with a language matching $\frac{\sum \mathcal{A B}}{\operatorname{poly}(a, b)} \cdot \frac{\sum \mathcal{B C}}{\operatorname{poly}(b, c)}$. Finally, there may be a final rule $X_{a \rightarrow c} \rightarrow c$ for nonterminal $X_{a \rightarrow c}$.

We can conclude that the languages $L\left(C_{a \rightarrow c}\right)$ satisfy the following system of language equations.

$$
L\left(C_{a \rightarrow c}\right)=\operatorname{end}\left(C_{a \rightarrow c}\right) \triangle \triangle_{C \rightarrow D E}\left(L\left(D_{a \rightarrow a}\right) \odot L\left(E_{a \rightarrow c}\right)\right) \triangle\left(L\left(D_{a \rightarrow c}\right) \odot L\left(E_{c \rightarrow c}\right)\right)
$$

Here, the summation happens over all rules $C \rightarrow D E$ for the nonterminal $C$ of the original $\mathrm{GF}(2)$-grammar, and $\operatorname{end}\left(C_{a \rightarrow c}\right)$ is defined as:

$$
\operatorname{end}\left(C_{a \rightarrow c}\right)=(\{c\} \text { or } \varnothing) \triangle \triangle_{C \rightarrow D E} L\left(D_{a \rightarrow b}\right) \odot L\left(E_{b \rightarrow c}\right)
$$

Here, the first "summand" depends on whether or not there is a rule $C_{a \rightarrow c} \rightarrow c$ in the new GF(2)-grammar. 
Consider the equations from the system (11) more closely. For all GF(2)-concatenations that appear in their right-hand sides, either the first multiplier is a subset of $a^{*}$, or the second is a subset of $c^{*}$. Therefore, we can replace all GF(2)-concatenations here satisfy the conditions of Lemma 3.1

$$
L\left(C_{a \rightarrow c}\right)=\operatorname{end}\left(C_{a \rightarrow c}\right) \triangle \triangle_{C \rightarrow D E}\left(L\left(D_{a \rightarrow a}\right) \odot_{\text {comm }} L\left(E_{a \rightarrow c}\right)\right) \triangle\left(L\left(D_{a \rightarrow c}\right) \odot_{\text {comm }} L\left(E_{c \rightarrow c}\right)\right)
$$

Denote $\operatorname{asSeries}\left(L\left(C_{a \rightarrow c}\right)\right)$ by $\operatorname{Center}(C)$, asSeries $\left(L\left(C_{a \rightarrow a}\right)\right)$ by $\operatorname{Left}(C)$, $\operatorname{asSeries}\left(L\left(C_{c \rightarrow c}\right)\right)$ by $\operatorname{Right}(C)$ and asSeries(end $\left.\left(C_{a \rightarrow c}\right)\right)$ by final $(C)$. By applying correspondence asSeries to the both sides of each equation of the system (13),

$$
\operatorname{Center}(C)=\operatorname{final}(C)+\sum_{C \rightarrow D E} \operatorname{Left}(D) \operatorname{Center}(E)+\operatorname{Center}(D) \operatorname{Right}(E)
$$

This system of equation can be interpeted as a system $\mathbb{F}_{2}[[a, b, c]]$-linear equations over variables Center $(C)=\operatorname{asSeries}\left(L\left(C_{a \rightarrow c}\right)\right)$ for every nonterminal $C$ of the original $\operatorname{GF}(2)$ grammar.

We will consider final $(C), \operatorname{Left}(C)$ and $\operatorname{Right}(C)$ to be the coefficients of said system. While we do not know their exact values, we know that final $(C)$ match the expression $\sum\left(\frac{\sum \mathcal{A B}}{\operatorname{poly}(a, b)}\right) \cdot\left(\frac{\sum \mathcal{B C}}{\operatorname{poly}(b, c)}\right)=\frac{\sum \mathcal{A B C}}{\operatorname{poly}(a, b) \operatorname{poly}(b, c)}$ by formula (12) and Theorem B.1, $\operatorname{Left}(C)$ is in $\mathcal{A}$, because it corresponds to a 2-automatic language over an alphabet $\{a\}$ and, similarly, $\operatorname{Right}(C)$ is in $\mathcal{C}$.

Let us say that the original GF(2)-grammar has $n$ nonterminals. Then the new GF(2)grammar has $n$ nonterminals of type $a \rightarrow c$. Denote the column-vector of values Center $(C)$ by $x$, and the column-vector of values of final $(C)$, listed in the same order, by $f$. Fix such numeration of nonterminals of the original GF(2)-grammar. Now, we can indice both rows and columns of $n \times n$ matrices by nonterminals of the original GF(2)-grammar.

Let $I$ be an identity $n \times n$ matrix and $A$ be a $n \times n$ matrix, where the cell on the intersection of $C$-th row and $E$-th column contains the sum Left $(D)$ over all rules $C \rightarrow D E$ of the original grammar:

$$
A_{C, E}:=\sum_{C \rightarrow D E} \operatorname{Left}(D)
$$

Similarly, let $B$ be $n \times n$ matrix with sum of $\operatorname{Right}(E)$ over all rules $C \rightarrow D E$ of the original grammar standing on the intersection of $C$-th row and $D$-th column (it would make more sense to call this matrix $C$, not $B$, but we already used letter $C$ for different purpose):

$$
B_{C, E}:=\sum_{C \rightarrow D E} \operatorname{Right}(E)
$$

Then, the system of equations (14) can be stated in the following compact matrix form: $x=f+(A+B) x$, or $(A+B+I) x=f$, which is the same.

As we showed above, the column-vector of Center $(C)$ values indeed is a solution to such a system. If we somehow establish that this system has only one solution, which 
can be expressed in relatively simple algebraic terms, we will get an expression for for $\operatorname{asSeries}\left(L\left(S_{a \rightarrow c}\right)\right)=\operatorname{Center}(S)$.

This system has exactly one solution if and only if $\operatorname{det}(A+B+I) \neq 0$. If $\operatorname{det}(A+B+I) \neq$ 0 , then, by Cramer's formula, each component of the solution, Center $(S)$, in particular, can be represented in the following form:

$$
\frac{\operatorname{det}(A+B+I, \text { but one of the columns was replaced by } f)}{\operatorname{det}(A+B+I)}
$$

Now, we still need to prove three things: that $\operatorname{det}(A+B+I)$ matches $\sum \mathcal{A C}$, that $\operatorname{det}\left(A+B+I\right.$, but one of the columns was replaced by $f$ matches $\frac{\sum \mathcal{A B C}}{\operatorname{poly}(a, b) \operatorname{poly}(b, c)}$, independently of replaced column, and, finally, that $\operatorname{det}(A+B+I)$ is not zero.

Each entry of $A+B+I$ match $\mathcal{A}+\mathcal{C}$, because of equations (15) and (16). Indeed, each entry of $A$ match $\sum \mathcal{A}=\mathcal{A}$, similarly, each component of $B$ matches $\mathcal{C}$, and entry of $I$ are zeroes and ones, which match both $\mathcal{A}$ and $\mathcal{C}$.

The determinant of the matrix with all entries matching $\mathcal{A}+\mathcal{C}$, matches $\sum \mathcal{A C}$. We have already proven that during the proof of Lemma B.1. The proof of $\operatorname{det}(A+B+I)$ being non-zero also comes from the same place verbatim.

Finally, determinant of $A+B+I$ with one column replaced by $f$ is something new, because entries of $f$ match rather complicated expression $\sum\left(\frac{\sum \mathcal{A B}}{\operatorname{poly}(a, b)}\right) \cdot\left(\frac{\sum B \mathcal{C}}{\operatorname{poly}(b, c)}\right)=$ $\frac{\sum \mathcal{A B C}}{\operatorname{poly}(a, b) \operatorname{poly}(b, c)}$. By using the formula for determinant with $n$ ! summands, we see that the determinant matches $\sum(\mathcal{A}+\mathcal{C}) \ldots(\mathcal{A}+\mathcal{C})\left(\frac{\sum \mathcal{A B C}}{\operatorname{poly}(a, b) \operatorname{poly}(b, c)}\right)(\mathcal{A}+\mathcal{C}) \ldots(\mathcal{A}+\mathcal{C})$. Here, in each summand one multiplier is complicated and the others are very simple. By expanding all brackets, the determinant matches $\sum \frac{\sum \mathcal{A B C}}{\operatorname{poly}(a, b) \operatorname{poly}(b, c)}$. By taking lowest common denominator of all fractions in the sum, the determinant matches $\frac{\sum \mathcal{A B C}}{\operatorname{poly}(a, b) \operatorname{poly}(b, c)}$.

Now, Center $(S)=L\left(S_{a \rightarrow c}\right)$ is not exactly the language described by the new $\mathrm{GF}(2)$-grammar, $L\left(S^{\prime}\right)=L\left(S_{a \rightarrow a}\right) \triangle L\left(S_{a \rightarrow b}\right) \triangle L\left(S_{a \rightarrow c}\right)$ is. However, $\operatorname{asSeries}\left(L\left(S^{\prime}\right)\right)=\operatorname{asSeries}\left(L\left(S_{a \rightarrow a}\right)\right)+\operatorname{asSeries}\left(L\left(S_{a \rightarrow b}\right)\right)+\operatorname{asSeries}\left(L\left(S_{a \rightarrow c}\right)\right)$. Therefore, series asSeries $(K)=\operatorname{arSeries}\left(L\left(S^{\prime}\right)\right) \operatorname{match} \mathcal{A}+\frac{\sum \mathcal{A B}}{\operatorname{poly}(a, b)}+\frac{\sum \mathcal{A B C}}{\operatorname{poly}(a, b) \operatorname{poly}(b, c) \sum \mathcal{A C}}=$ $\sum \mathcal{A B C}$ $\overline{\text { poly }(a, b) \text { poly }(b, c) \sum \mathcal{A C}}$. The last equivalence holds, because the first two summands are simple and are "absorbed" by complicated third summand.

\section{C.2 Algebraic manipulations}

We will establish the following theorem: 
Theorem C.1. Let $L$ be a subset of $a^{*} b^{*} c^{*}$ described by a GF(2)-grammar. Then, the formal power series asSeries $(L)$ match algebraic expression $\frac{\sum \mathcal{A B C}}{\operatorname{poly}(a, b) \operatorname{poly}(b, c) \sum \mathcal{A C}}$.

By Lemma C.1, it is enough to prove the following lemma:

Lemma C.2. The algebraic expressions $\frac{\sum \mathcal{A B C}}{\operatorname{poly}(a, b) \operatorname{poly}(b, c) \sum \mathcal{A C}} \quad$ and $\frac{\sum \mathcal{A B C}}{\operatorname{poly}(a, b) \operatorname{poly}(b, c) \operatorname{poly}(a, c)}$ are equivalent.

Proof. The proof is much simpler than the proof of Theorem B.1, because we can use it now.

The second expression is not stronger than the first, because poly $(a, c)$ is not stronger than $\sum \mathcal{A C}$.

On the other hand, we already know that the expression $\frac{\sum \mathcal{A C}}{\sum \mathcal{A C}}$ is not stronger than $\frac{\sum \mathcal{A C}}{\text { poly }(a, c)}$, because we needed that to prove Theorem B.1. Therefore, the expression $\frac{\sum \mathcal{A B C}}{\operatorname{poly}(a, b) \operatorname{poly}(b, c) \sum \mathcal{A C}}$ is not stronger than $\frac{\sum \mathcal{A B C} \cdot \sum \mathcal{A C}}{\operatorname{poly}(a, b) \operatorname{poly}(b, c) \operatorname{poly}(a, c)}$. In the last expression, the multiplier $\sum \mathcal{A C}$ can be "absorbed" into $\sum \mathcal{A B C}$, giving us exactly the expression $\frac{\sum \mathcal{A B C}}{\operatorname{poly}(a, b) \operatorname{poly}(b, c) \operatorname{poly}(a, c)}$.

Remark. In a very similar way, with induction over the number $k$ of letters in the alphabet, we can prove the following result: for $K \subset a_{1}^{*} a_{2}^{*} \ldots a_{k}^{*}$, the corresponding power series $\operatorname{asSeries}(K)$ match the expression $\frac{\sum \prod_{i=1}^{n} \mathcal{A}_{i}}{\prod_{1 \leqslant i<j \leqslant n} \operatorname{poly}\left(a_{i}, a_{j}\right)}$, where $\mathcal{A}_{i}$ is the set of algebraic formal power series of variable $a_{i}$. 